\title{
Removahedral congruences versus permutree congruences
}

\author{
Doriann Albertin \\ LIGM, Université Gustave Eiffel \\ Champs-sur-Marne, France \\ doriann.albertin@u-pem.fr
}

\author{
Vincent Pilaud* \\ CNRS \& LIX, École Polytechnique \\ Palaiseau, France \\ vincent.pilaud@lix.polytechnique.fr
}

\author{
Julian Ritter* \\ LIX, École Polytechnique \\ Palaiseau, France \\ julian.ritter@polytechnique.edu
}

Submitted: Feb 1, 2021; Accepted: Sep 23, 2021; Published: Oct 22, 2021

(C) The authors. Released under the CC BY-ND license (International 4.0).

\begin{abstract}
The associahedron is classically constructed as a removahedron, i.e. by deleting inequalities in the facet description of the permutahedron. This removahedral construction extends to all permutreehedra (which interpolate between the permutahedron, the associahedron and the cube). Here, we investigate removahedra constructions for all quotientopes (which realize the lattice quotients of the weak order). On the one hand, we observe that the permutree fans are the only quotient fans realized by a removahedron. On the other hand, we show that any permutree fan can be realized by a removahedron constructed from any realization of the braid fan. Our results finally lead to a complete description of the type cones of the permutree fans.
\end{abstract}

Mathematics Subject Classifications: 52B11, 52B12, 03G10, 06B10

\section{Introduction}

This paper deals with particular polytopal realizations of quotient fans of lattice congruences of the weak order. The prototypes of such polytopes are the classical permutahedron Perm ${ }_{n}$ realizing the weak order on permutations and the classical associahedron Asso ${ }_{n}$

\footnotetext{
*Supported by the French ANR (grants CAPPS 17 CE40 0018 and CHARMS 19 CE40 0017).
} 
realizing the Tamari lattice on binary trees. These two polytopes belong to the family of permutreehedra realizing the rotation lattice on permutrees, which play a fundamental role in this paper. Permutrees were introduced in [PP18] to generalize and interpolate between permutations and binary trees, and explain the combinatorial, geometric and algebraic similarities between them. They were inspired by Cambrian trees [CP17, LP18] which provide a combinatorial model to the type $A$ Cambrian lattices of [Rea06]. As the classical construction of the associahedron due to [SS93, Lod04] and its generalization to all Cambrian associahedra by [HL07], all permutreehedra are obtained by deleting inequalities in the facet description of the permutahedron Perm ${ }_{n}$. Such polytopes are called removahedra, and were studied in the context of graph associahedra in [Pil17].

In general, any lattice congruence $\equiv$ of the weak order on $\mathfrak{S}_{n}$ defines a quotient fan $\mathcal{F}_{\equiv}$ obtained by gluing together the chambers of the braid fan corresponding to permutations in the same congruence class [Rea05]. This quotient fan $\mathcal{F}_{\equiv}$ was recently proven to be the normal fan of a polytope $P_{\equiv}$ called quotientope [PS19, PPR20]. As their normal fans all refine the braid fan, quotientopes belong to the class of deformed permutahedra studied in [Pos09, PRW08] (we prefer the name "deformed permutahedra" rather than "generalized permutahedra" as there are many generalizations of permutahedra). All deformed permutahedra are obtained from the permutahedron Perm ${ }_{n}$ by moving facets without "passing a vertex" (in the sense of [Pos09]). Observe that not all deformed permutahedra are removahedra, since it is sometimes inevitable to move facets, not only to remove them.

The construction of [PP18] for permutreehedra and the construction of [PS19] for quotientopes seem quite different. The polytopes resulting from the former construction

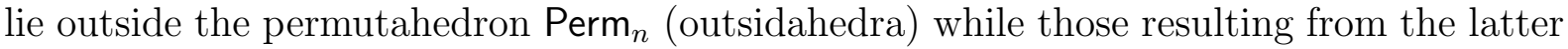

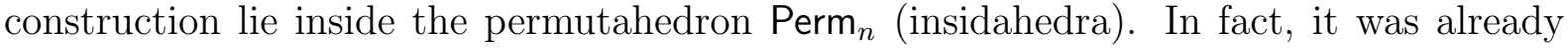
observed in [PS19, Rem. 12] that the quotient fans of some lattice congruences cannot be realized by a removahedron. The first contribution of this paper is to show the following strong dichotomy between the lattices congruences of the weak order regarding realizability by removahedra.

Theorem 1. Let $\equiv$ be a lattice congruence of the weak order on $\mathfrak{S}_{n}$. Then

(i) if $\equiv$ is not a permutree congruence, then the quotient fan $\mathcal{F}_{\equiv}$ is not the normal fan of a removahedron,

(ii) if $\equiv$ is a permutree congruence, then the quotient fan $\mathcal{F}_{\equiv}$ is the normal fan of a polytope obtained by deleting inequalities in the facet description of any polytope realizing the braid fan (not only the classical permutahedron Perm $_{n}$ ).

This statement is based on the understanding of the inequalities governing the facet heights that ensure to obtain a polytopal realization of the quotient fan. These inequalities, given by pairs of adjacent cones of the fan and known as wall-crossing inequalities, define the space of all realizations of the fan. This space of realizations is an open polyhedral cone called type cone and studied by [McM73], and its closure is also known as 
the deformation cone [Pos09, PRW08]. For instance, the deformation cone of the permutahedron Perm $_{n}$ is the space of submodular functions, and corresponds to all deformed permutahedra [Pos09]. The main contribution of this paper is the facet description of the type cone of any permutree fan, thus providing a complete description of all polytopal realizations of the permutree fans. This description requires three steps: for the $\delta$-permutree fan $\mathcal{F}_{\delta}$ corresponding to a decoration $\delta$, we provide purely combinatorial descriptions (only in terms of $\delta$ ) for

- the subsets of $[n]$ which correspond to rays of $\mathcal{F}_{\delta}$ (Proposition 32 ),

- the pairs of subsets of $[n]$ which correspond to exchangeables rays of $\mathcal{F}_{\delta}$ (Proposition 39),

- the pairs of subsets of $[n]$ which correspond to facets of the type cone of $\mathcal{F}_{\delta}$ (Proposition 45).

From this facet description, we derive summation formulas for the number of facets of the type cones of permutree fans, leading to a characterization of the permutree fans whose type cone is simplicial. As advocated in [PPPP19], this property is interesting because it leads on the one hand to a simple description of all polytopal realizations of the fan in the kinematic space [AHBHY18], and on the other hand to canonical Minkowski sum decompositions of these realizations.

This paper opens the door to a description of the type cone of the quotient fan for any lattice congruence of the weak order on $\mathfrak{S}_{n}$, not only for permutree congruences. Preliminary computations however indicate that the combinatorics of the facet description of the type cone of an arbitrary quotient fan is much more intricated than that of permutree fans.

The paper is organized as follows. Section 2 provides a recollection of all material needed in the paper, including polyhedral geometry and type cones (Section 2.1), lattice quotients of the weak order (Section 2.2), deformed permutahedra and removahedra (Section 2.3) and permutrees (Section 2.4). Section 3 is devoted to the proof of Theorem 1. Finally, the type cones of all permutree fans are described in Section 4.

\section{Preliminaries}

We start with preliminaries on polyhedral geometry, type cones, braid arrangements, quotient fans, shards, deformed permutahedra, removahedra and permutrees. The presentation is largely inspired by the papers [PPPP19, PS19, PP18] and we reproduce here some of their pictures.

\subsection{Polyhedral geometry and type cones}

We start with basic notions of polyhedral geometry (see G. Ziegler's textbook [Zie98]) and a short introduction to type cones (see the original work of P. McMullen [McM73] or their recent application to $\boldsymbol{g}$-vector fans in [PPPP19]). 


\subsubsection{Polyhedral geometry}

A hyperplane $H \subset \mathbb{R}^{n}$ is a supporting hyperplane of a set $X \subset \mathbb{R}^{n}$ if $H \cap X \neq \varnothing$ and $X$ is contained in one of the two closed half-spaces of $\mathbb{R}^{n}$ defined by $H$.

We denote by $\mathbb{R}_{\geqslant 0} \boldsymbol{R}:=\left\{\sum_{\boldsymbol{r} \in \boldsymbol{R}} \lambda_{\boldsymbol{r}} \boldsymbol{r} \mid \lambda_{\boldsymbol{r}} \in \mathbb{R}_{\geqslant 0}\right\}$ the positive span of a set $\boldsymbol{R}$ of vectors of $\mathbb{R}^{n}$. A (polyhedral) cone is a subset of $\mathbb{R}^{n}$ defined equivalently as the positive span of finitely many vectors or as the intersection of finitely many closed linear halfspaces. Its faces are its intersections with its supporting linear hyperplanes, and its rays (resp. facets) are its dimension 1 (resp. codimension 1) faces. A cone is simplicial if it is generated by a set of linearly independent vectors.

A (polyhedral) fan $\mathcal{F}$ is a collection of cones which are closed under faces (if $C \in \mathcal{F}$ and $F$ is a face of $C$, then $F \in \mathcal{F}$ ) and intersect properly (if $C, D \in \mathcal{F}$, then $C \cap D$ is a face of both $C$ and $D$ ). The chambers (resp. walls, resp. rays) of $\mathcal{F}$ are its codimension 0 (resp. codimension 1, resp. dimension 1) cones. The fan $\mathcal{F}$ is simplicial if all its cones are, complete if the union of its cones covers the ambient space $\mathbb{R}^{n}$, and essential if it contains the cone $\{\boldsymbol{0}\}$. Note that every complete fan is the product of an essential fan with its lineality space (the largest linear subspace contained in all cones of $\mathcal{F}$ ). Given two fans $\mathcal{F}, \mathcal{G}$ in $\mathbb{R}^{n}$, we say that $\mathcal{F}$ refines $\mathcal{G}$ (and that $\mathcal{G}$ coarsens $\mathcal{F}$ ) if every cone of $\mathcal{G}$ is a union of cones of $\mathcal{F}$. In a simplicial fan, we say that two maximal cones are adjacent if they share a facet, and that two rays are exchangeable if they belong to two adjacent cones but not to their common facet.

A polytope is a subset of $\mathbb{R}^{n}$ defined equivalently as the convex hull of finitely many points or as a bounded intersection of finitely many closed affine halfspaces. Its faces are its intersections with its supporting affine hyperplanes, and its vertices (resp. edges, resp. facets) are its dimension 0 (resp. dimension 1, codimension 1) faces. The normal cone of a face $F$ of a polytope $P$ is the cone generated by the outer normal vectors of the facets of $P$ containing $F$. The normal fan of $P$ is the fan formed by the normal cones of all faces of $P$.

\subsubsection{Type cones}

Fix an essential complete simplicial fan $\mathcal{F}$ in $\mathbb{R}^{n}$ with $N$ rays. Let $\boldsymbol{G}$ be the $N \times n$ matrix whose rows are (representative vectors for) the rays of $\mathcal{F}$. For any vector $\boldsymbol{h} \in \mathbb{R}^{N}$, we define the polytope $P_{\boldsymbol{h}}:=\left\{\boldsymbol{x} \in \mathbb{R}^{n} \mid \boldsymbol{G} \boldsymbol{x} \leqslant \boldsymbol{h}\right\}$. In other words, $P_{\boldsymbol{h}}$ has an inequality $\langle\boldsymbol{r} \mid \boldsymbol{x}\rangle \leqslant \boldsymbol{h}_{\boldsymbol{r}}$ for each ray $\boldsymbol{r}$ of $\mathcal{F}$, where $\boldsymbol{h}_{\boldsymbol{r}}$ denotes the coordinate of $\boldsymbol{h}$ corresponding to $\boldsymbol{r}$. Note that $\mathcal{F}$ is not necessarily the normal fan of $P_{\boldsymbol{h}}$. The vectors $\boldsymbol{h}$ for which this holds are characterized by the following classical statement. It already appeared in the study of Minkowski summands of polytopes [Mey74, McM73], and in the theory of secondary polytopes [GKZ08], see also [DRS10]. We present here a convenient formulation from [CFZ02, Lem. 2.1]. 
Proposition 2. Let $\mathcal{F}$ be an essential complete simplicial fan in $\mathbb{R}^{n}$ and let $\boldsymbol{G}$ be the $N \times n$-matrix whose rows are the rays of $\mathcal{F}$. Then the following are equivalent for any vector $\boldsymbol{h} \in \mathbb{R}^{N}$ :

1. The fan $\mathcal{F}$ is the normal fan of the polytope $P_{\boldsymbol{h}}:=\left\{\boldsymbol{x} \in \mathbb{R}^{n} \mid \boldsymbol{G} \boldsymbol{x} \leqslant \boldsymbol{h}\right\}$.

2. For any two adjacent chambers $\mathbb{R}_{\geqslant 0} \boldsymbol{R}$ and $\mathbb{R}_{\geqslant 0} \boldsymbol{S}$ of $\mathcal{F}$ with $\boldsymbol{R} \backslash\{\boldsymbol{r}\}=\boldsymbol{S} \backslash\{\boldsymbol{s}\}$,

$$
\alpha \boldsymbol{h}_{\boldsymbol{r}}+\beta \boldsymbol{h}_{\boldsymbol{s}}+\sum_{\boldsymbol{t} \in \boldsymbol{R} \cap \boldsymbol{S}} \gamma_{\boldsymbol{t}} \boldsymbol{h}_{\boldsymbol{t}}>0,
$$

where

$$
\alpha \boldsymbol{r}+\beta \boldsymbol{s}+\sum_{\boldsymbol{t} \in \boldsymbol{R} \cap \boldsymbol{S}} \gamma_{\boldsymbol{t}} \boldsymbol{t}=0
$$

is the unique (up to rescaling) linear dependence with $\alpha, \beta>0$ between the rays of $\boldsymbol{R} \cup \boldsymbol{S}$.

The inequalities in this statement are called wall-crossing inequalities. For convenience, let us denote by $\alpha_{\boldsymbol{R}, \boldsymbol{S}}(\boldsymbol{t})$ the coefficient of $\boldsymbol{t}$ in the unique linear dependence between the rays of $\boldsymbol{R} \cup \boldsymbol{S}$ such that $\alpha_{\boldsymbol{R}, \boldsymbol{S}}(\boldsymbol{r})+\alpha_{\boldsymbol{R}, \boldsymbol{S}}(\boldsymbol{s})=2$, so that the inequality above rewrites as $\sum_{\boldsymbol{t} \in \boldsymbol{R} \cup \boldsymbol{S}} \alpha_{\boldsymbol{R}, \boldsymbol{S}}(\boldsymbol{t}) \boldsymbol{h}_{\boldsymbol{t}}>0$.

When considering the question of the realizability of a complete simplicial fan $\mathcal{F}$ by a polytope, it is natural to consider all possible realizations of this fan, as was done by P. McMullen in [McM73]. The type cone of $\mathcal{F}$ is the cone

$$
\begin{aligned}
\mathbb{T} C(\mathcal{F}) & :=\left\{\boldsymbol{h} \in \mathbb{R}^{N} \mid \mathcal{F} \text { is the normal fan of } P_{\boldsymbol{h}}\right\} \\
& =\left\{\boldsymbol{h} \in \mathbb{R}^{N} \mid \sum_{\boldsymbol{t} \in \boldsymbol{R} \cup \boldsymbol{S}} \alpha_{\boldsymbol{R}, \boldsymbol{S}}(\boldsymbol{t}) \boldsymbol{h}_{\boldsymbol{t}}>0 \begin{array}{l}
\text { for any adjacent chambers } \\
\mathbb{R}_{\geqslant 0} \boldsymbol{R} \text { and } \mathbb{R}_{\geqslant 0} \boldsymbol{S} \text { of } \mathcal{F}
\end{array}\right\} .
\end{aligned}
$$

The type cone $\mathbb{T} \mathbb{C}(\mathcal{F})$ is an open cone. We denote by $\overline{\mathbb{T}}(\mathcal{F})$ the closure of $\mathbb{T} \mathbb{C}(\mathcal{F})$, and still call it the type cone of $\mathcal{F}$. It is the closed polyhedral cone defined by the inequalities $\sum_{\boldsymbol{t} \in \boldsymbol{R} \cup \boldsymbol{S}} \alpha_{\boldsymbol{R}, \boldsymbol{S}}(\boldsymbol{t}) \boldsymbol{h}_{\boldsymbol{t}} \geqslant 0$ for any adjacent chambers $\mathbb{R}_{\geqslant 0} \boldsymbol{R}$ and $\mathbb{R}_{\geqslant 0} \boldsymbol{S}$. It describes all polytopes $P_{\boldsymbol{h}}$ whose normal fans coarsen the fan $\mathcal{F}$. If $\mathcal{F}$ is the normal fan of the polytope $P$, then $\overline{\mathbb{T C}}(\mathcal{F})$ is also known as the deformation cone of $P$, see [Pos09, PRW08]. We use $\overline{\mathbb{T} C}(\mathcal{F})$ rather than $\mathbb{T} \mathbb{C}(\mathcal{F})$ when we want to speak about the facets of $\overline{\mathbb{T} C}(\mathcal{F})$.

Also observe that the lineality space of the type cone $\overline{\mathbb{T} C}(\mathcal{F})$ has dimension $n$ (it is invariant by translation in $\left.G \mathbb{R}^{n}\right)$. In particular, the type cone $\overline{\mathbb{T} C}(\mathcal{F})$ is simplicial when it has $N-n-1$ facets. While very particular, the fans for which the type cone is simplicial are very interesting as all their polytopal realizations can be described as follows.

Proposition 3 ([PPPP19, Coro. 1.11]). Let $\mathcal{F}$ be an essential complete simplicial fan in $\mathbb{R}^{n}$ with $N$ rays, such that the type cone $\overline{\mathbb{T}} \mathbb{C}(\mathcal{F})$ is simplicial. Let $\boldsymbol{K}$ be the $(N-n) \times N$ matrix whose rows are the inner normal vectors of the facets of $\overline{\mathbb{T}}(\mathcal{F})$. Then the polytope

$$
Q(\boldsymbol{u}):=\left\{\boldsymbol{z} \in \mathbb{R}_{\geqslant 0}^{N} \mid \boldsymbol{K} \boldsymbol{z}=\boldsymbol{u}\right\}
$$

is a realization of the fan $\mathcal{F}$ for any positive vector $\boldsymbol{u} \in \mathbb{R}_{>0}^{N-n}$. Moreover, the polytopes $Q(\boldsymbol{u})$ for $\boldsymbol{u} \in \mathbb{R}_{>0}^{N-n}$ describe all polytopal realizations of $\mathcal{F}$. 
In this paper, we shall also use a non-simplicial version of Proposition 2, see [Mey74].

Proposition 4. Let $\mathcal{F}$ be an essential complete (non necessarily simplicial) fan in $\mathbb{R}^{n}$ and $\boldsymbol{G}$ be the $N \times n$-matrix whose rows are the rays of $\mathcal{F}$. Then the following are equivalent for any $\boldsymbol{h} \in \mathbb{R}^{N}$ :

1. The fan $\mathcal{F}$ is the normal fan of the polytope $P_{\boldsymbol{h}}:=\left\{\boldsymbol{x} \in \mathbb{R}^{n} \mid \boldsymbol{G} \boldsymbol{x} \leqslant \boldsymbol{h}\right\}$.

2. The coordinates of $\boldsymbol{h}$ satisfy the following equalities and inequalities:

- for any chamber $\mathbb{R}_{\geqslant 0} \boldsymbol{R}$ of $\mathcal{F}$ and any linear dependence $\sum_{\boldsymbol{r} \in \boldsymbol{R}} \gamma_{\boldsymbol{r}} \boldsymbol{r}=0$ among the rays of $\boldsymbol{R}$, we have $\sum_{\boldsymbol{r} \in \boldsymbol{R}} \gamma_{\boldsymbol{r}} \boldsymbol{h}_{\boldsymbol{r}}=0$,

- for any two adjacent chambers $\mathbb{R}_{\geqslant 0} \boldsymbol{R}$ and $\mathbb{R}_{\geqslant 0} \boldsymbol{S}$ of $\mathcal{F}$, any rays $\boldsymbol{r} \in \boldsymbol{R} \backslash \boldsymbol{S}$ and $\boldsymbol{s} \in \boldsymbol{S} \backslash \boldsymbol{R}$, and any linear dependence $\alpha \boldsymbol{r}+\beta \boldsymbol{s}+\sum_{\boldsymbol{t} \in \boldsymbol{R} \cap \boldsymbol{S}} \gamma_{\boldsymbol{t}} \boldsymbol{t}=0$ among the rays $\{\boldsymbol{r}, \boldsymbol{s}\} \cup(\boldsymbol{R} \cap \boldsymbol{S})$ with $\alpha, \beta>0$, we have $\alpha \boldsymbol{h}_{\boldsymbol{r}}+\beta \boldsymbol{h}_{\boldsymbol{s}}+\sum_{\boldsymbol{t} \in \boldsymbol{R} \cap \boldsymbol{S}} \gamma_{\boldsymbol{t}} \boldsymbol{h}_{\boldsymbol{t}}>0$.

\subsection{Geometry of lattice quotients of the weak order}

We now recall the combinatorial and geometric toolbox to deal with lattice quotients of the weak order on $\mathfrak{S}_{n}$. The presentation and pictures are borrowed from [PS19].

\subsubsection{Weak order, braid fan, and permutahedron}

Let $\mathfrak{S}_{n}$ denote the set of permutations of the set $[n]:=\{1, \ldots, n\}$. The inversion set of a permutation $\sigma \in \mathfrak{S}_{n}$ is $\operatorname{inv}(\sigma):=\left\{\left(\sigma_{i}, \sigma_{j}\right) \mid 1 \leqslant i<j \leqslant n\right.$ and $\left.\sigma_{i}>\sigma_{j}\right\}$. We consider the weak order on $\mathfrak{S}_{n}$ defined by $\sigma \leqslant \tau \Longleftrightarrow \operatorname{inv}(\sigma) \subseteq \operatorname{inv}(\tau)$. See Figure 1 (left). This order can be interpreted geometrically on the braid fan $\mathcal{F}_{n}$ or the permutahedron Perm ${ }_{n}$ defined below.

The braid arrangement is the set $\mathcal{H}_{n}$ of linear hyperplanes $\left\{\boldsymbol{x} \in \mathbb{R}^{n} \mid \boldsymbol{x}_{i}=\boldsymbol{x}_{j}\right\}$ for all $1 \leqslant i<j \leqslant n$. As all hyperplanes of $\mathcal{H}_{n}$ contain the line $\mathbb{R} \mathbf{1}:=\mathbb{R}(1,1, \ldots, 1)$, we restrict
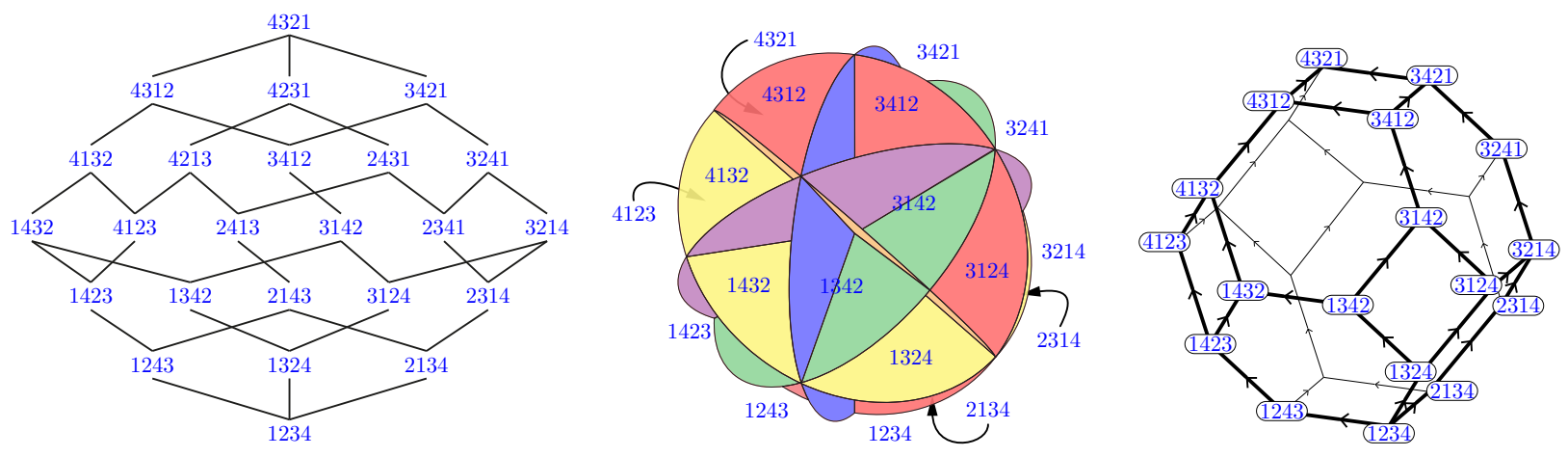

Figure 1: The Hasse diagram of the weak order on $\mathfrak{S}_{4}$ (left) can be seen as the dual graph of the braid fan $\mathcal{F}_{4}$ (middle) or as the graph of the permutahedron Perm 4 (right). [PS19, Fig. 1] 
to the hyperplane $\mathbb{H}:=\left\{\boldsymbol{x} \in \mathbb{R}^{n} \mid \sum_{i \in[n]} \boldsymbol{x}_{i}=0\right\}$. The hyperplanes of $\mathcal{H}_{n}$ divide $\mathbb{H}$ into chambers, which are the maximal cones of a complete simplicial fan $\mathcal{F}_{n}$, called braid fan. It has

- a chamber $C(\sigma):=\left\{\boldsymbol{x} \in \mathbb{H} \mid \boldsymbol{x}_{\sigma_{1}} \leqslant \boldsymbol{x}_{\sigma_{2}} \leqslant \ldots \leqslant \boldsymbol{x}_{\sigma_{n}}\right\}$ for each permutation $\sigma$ of $\mathfrak{S}_{n}$,

- a ray $C(I):=\left\{\boldsymbol{x} \in \mathbb{H} \mid \boldsymbol{x}_{i_{1}}=\cdots=\boldsymbol{x}_{i_{p}} \leqslant \boldsymbol{x}_{j_{1}}=\cdots=\boldsymbol{x}_{j_{n-p}}\right\}$ for each $\varnothing \neq I \subsetneq[n]$, where $I=\left\{i_{1}, \ldots, i_{p}\right\}$ and $[n] \backslash I=\left\{j_{1}, \ldots, j_{n-p}\right\}$. When needed, we use the representative vector $\boldsymbol{r}(I):=|I| \mathbf{1}-n \mathbf{1}_{I}$ in $C(I)$, where $\mathbf{1}:=\sum_{i \in[n]} \boldsymbol{e}_{i}$ and $\mathbf{1}_{I}:=\sum_{i \in I} \boldsymbol{e}_{i}$. (Note that the advantage of $\boldsymbol{r}(I)$ over $\mathbf{1}_{I}$ is that it belongs to the hyperplane $\mathbb{H}$ and leads to a simple formula for the support function of the classical permutahedron.)

The chamber $C(\sigma)$ has rays $C(\sigma([k]))$ for $k \in[n]$. See Figures 1 (middle), 3 (left) and 4 (left) where the chambers are labeled in blue and the rays are labeled in red.

The permutahedron is the polytope Perm $_{n}$ defined equivalently as:

- the convex hull of the points $\sum_{i \in[n]} i \boldsymbol{e}_{\sigma_{i}}$ for all permutations $\sigma \in \mathfrak{S}_{n}$,

- the intersection of the hyperplane $\mathbf{H}:=\left\{\boldsymbol{x} \in \mathbb{R}^{n} \mid \sum_{i \in[n]} \boldsymbol{x}_{i}=\left(\begin{array}{c}n+1 \\ 2\end{array}\right)\right\}$ with the halfspaces $\left\{\boldsymbol{x} \in \mathbb{R}^{n} \mid \sum_{i \in I} \boldsymbol{x}_{i} \geqslant\left(\begin{array}{c}|I|+1 \\ 2\end{array}\right)\right\}$ for all proper subsets $\varnothing \neq I \subsetneq[n]$.

See Figure 1 (right). Note that this standard facet description is equivalent to the inequalities $\langle\boldsymbol{r}(I) \mid \boldsymbol{x}\rangle \leqslant n|I|(n-|I|) / 2$ for all proper subsets $\varnothing \neq I \subsetneq[n]$, which matches the conventions of Section 2.1.2.

The normal fan of the permutahedron Perm $_{n}$ is the braid fan $\mathcal{F}_{n}$. The Hasse diagram of the weak order on $\mathfrak{S}_{n}$ can be seen geometrically as the dual graph of the braid fan $\mathcal{F}_{n}$, or the graph of the permutahedron Perm $n$, when oriented in the linear direction $\boldsymbol{\alpha}:=\sum_{i \in[n]}(2 i-n-1) \boldsymbol{e}_{i}=(-n+1,-n+3, \ldots, n-3, n-1)$. See Figure 1.

\subsubsection{Lattice congruences and quotient fans}

A lattice congruence of a lattice $(L, \leqslant, \wedge, \vee)$ is an equivalence relation on $L$ that respects the meet and the join operations, i.e. such that $x \equiv x^{\prime}$ and $y \equiv y^{\prime}$ implies $x \wedge y \equiv x^{\prime} \wedge y^{\prime}$ and $x \vee y \equiv x^{\prime} \vee y^{\prime}$. It defines a lattice quotient $L / \equiv$ on the congruence classes of $\equiv$ where $X \leqslant Y$ if and only if there exist $x \in X$ and $y \in Y$ such that $x \leqslant y$, and $X \wedge Y$ (resp. $X \vee Y$ ) is the congruence class of $x \wedge y$ (resp. $x \vee y$ ) for any $x \in X$ and $y \in Y$.

Example 5. The prototype lattice congruence of the weak order is the sylvester congruence $\equiv_{\text {sylv }}$, see [LR98, HNT05]. Its congruence classes are the fibers of the binary search tree insertion algorithm, or equivalently the sets of linear extensions of binary trees (labeled in inorder and considered as posets oriented from bottom to top). It can also be seen as the transitive closure of the rewriting rule $U i k V j W \equiv_{\text {sylv }} U k i V j W$ for some letters $i<j<k$ and words $U, V, W$ on $[n]$. The quotient of the weak order by the sylvester congruence is (isomorphic to) the classical Tamari lattice [Tam51], whose elements are the binary trees on $n$ nodes and whose cover relations are rotations in binary trees. The sylvester congruence and the Tamari lattice are illustrated in Figure 2 

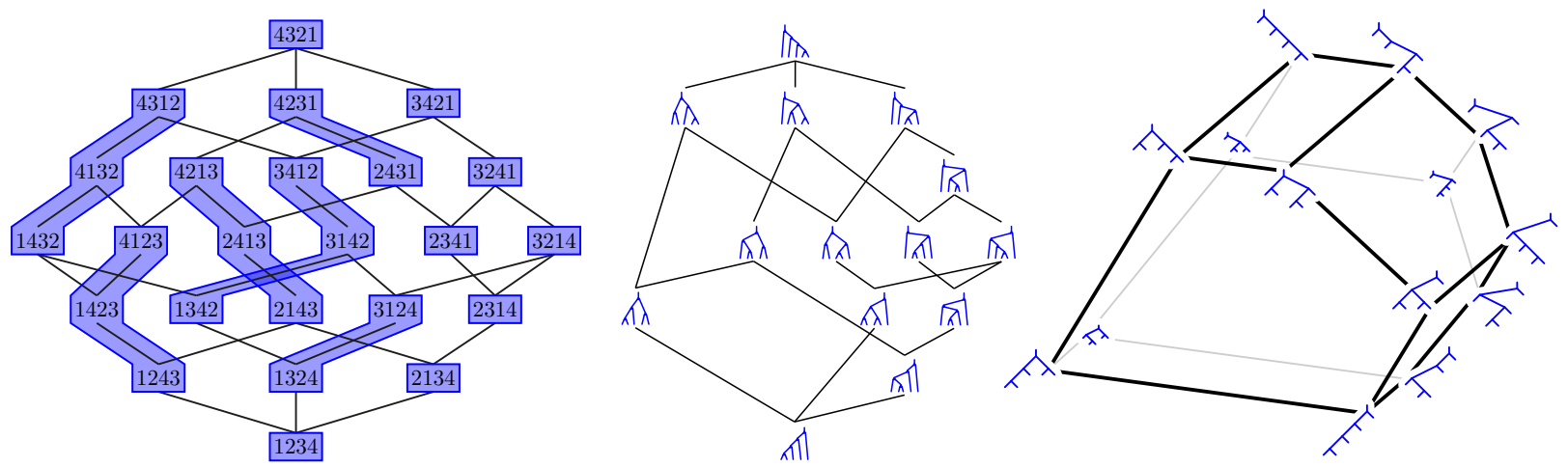

Figure 2: The quotient of the weak order by the sylvester congruence $\equiv_{\text {sylv }}$ (left) is the Tamari lattice (middle) and is realized by J.-L. Loday's associahedron (right). [PS19, Fig. 2]

for $n=4$. We will use the sylvester congruence and the Tamari lattice as a familiar example throughout the paper.

Lattice congruences naturally yield quotient fans, which turn out to be polytopal.

Theorem 6 ([Rea05]). Any lattice congruence $\equiv$ of the weak order on $\mathfrak{S}_{n}$ defines a complete fan $\mathcal{F}_{\equiv}$, called quotient fan, whose chambers are obtained gluing together the chambers $C(\sigma)$ of the braid fan $\mathcal{F}_{n}$ corresponding to permutations $\sigma$ that belong to the same congruence class of $\equiv$.

Theorem 7 ([PS19]). For any lattice congruence $\equiv$ of the weak order on $\mathfrak{S}_{n}$, the quotient fan $\mathcal{F}_{\equiv}$ is the normal fan of a polytope $P_{\equiv}$, called quotientope.

By construction, the Hasse diagram of the quotient of the weak order by $\equiv$ is given by the dual graph of the quotient fan $\mathcal{F}_{\equiv}$, or by the graph of the quotientope $P_{\equiv}$, oriented in the direction $\boldsymbol{\alpha}$.

Example 8. For the sylvester congruence $\equiv_{\text {sylv }}$ of Example 5 , the quotient fan $\mathcal{F}_{\text {sylv }}$ has

- a chamber $C(T)=\left\{\boldsymbol{x} \in \mathbb{H} \mid \boldsymbol{x}_{i} \leqslant \boldsymbol{x}_{j}\right.$ if $i$ is a descendant of $j$ in $\left.T\right\}$ for each binary tree $T$,

- a ray $C(I)$ for each proper interval $I=[i, j] \subsetneq[n]$.

Figures 3 and 4 (right) illustrate the quotient fans $\mathcal{F}_{\text {sylv }}$ for $n=3$ and $n=4$. The quotient fan $\mathcal{F}_{\text {sylv }}$ is the normal fan of the classical associahedron Asso $_{n}$ defined equivalently as:

- the convex hull of the points $\sum_{j \in[n]} \ell(T, j) r(T, j) \boldsymbol{e}_{j}$ for all binary trees $T$ on $n$ nodes, where $\ell(T, j)$ and $r(T, j)$ respectively denote the numbers of leaves in the left and right subtrees of the node $j$ of $T$ (labeled in inorder), see [Lod04],

- the intersection of the hyperplane $\mathbf{H}:=\left\{\boldsymbol{x} \in \mathbb{R}^{n} \mid \sum_{i \in[n]} \boldsymbol{x}_{i}=\left(\begin{array}{c}n+1 \\ 2\end{array}\right)\right\}$ with the halfspaces $\left\{\boldsymbol{x} \in \mathbb{R}^{n} \mid \sum_{i \leqslant k \leqslant j} \boldsymbol{x}_{k} \geqslant\left(\begin{array}{c}j-i+2 \\ 2\end{array}\right)\right\}$ for all $1 \leqslant i \leqslant j \leqslant n$, see [SS93].

See Figure 2 (right). 


\subsubsection{Shards}

An alternative description of the quotient fan $\mathcal{F}_{\equiv}$ defined in Theorem 6 is given by its walls, each of which can be seen as the union of some preserved walls of the braid arrangement. The conditions in the definition of lattice congruences impose strong constraints on the set of preserved walls: deleting some walls forces to delete others. Shards were introduced by N. Reading in [Rea03] (see also [Rea16b, Rea16a]) to understand the possible sets of preserved walls.

For any $1 \leqslant i<j \leqslant n$, let $[i, j]:=\{i, \ldots, j\}$ and $] i, j[:=\{i+1, \ldots, j-1\}$. For any $S \subseteq] i, j[$, the shard $\Sigma(i, j, S)$ is the cone

$$
\Sigma(i, j, S):=\left\{\boldsymbol{x} \in \mathbb{R}^{n} \mid \boldsymbol{x}_{i}=\boldsymbol{x}_{j}, \boldsymbol{x}_{i} \geqslant \boldsymbol{x}_{h} \text { for all } h \in S, \boldsymbol{x}_{i} \leqslant \boldsymbol{x}_{k} \text { for all } k \in\right] i, j[\backslash S\} .
$$

The length of $\Sigma(i, j, S)$ is $j-i$. Denote by $\Sigma_{n}:=\{\Sigma(i, j, S) \mid 1 \leqslant i<j \leqslant n$ and $S \subseteq] i, j[\}$ the set of all shards of $\mathcal{H}_{n}$.

Throughout the paper, we use a convenient notation for shards borrowed from N. Reading's work on arc diagrams [Rea15]: we consider $n$ dots on the horizontal axis, and we represent the shard $\Sigma(i, j, S)$ by an arc joining the $i$ th dot to the $j$ th dot and passing above (resp. below) the $k$ th dot when $k \in S$ (resp. when $k \notin S$ ). For instance, the arc $\curvearrowright \bullet$ represents the shard $\Sigma(1,4,\{2\})$. We say that $\Sigma(i, j, S)$ is an up (resp. down) shard if the corresponding arc passes above (resp. below) all points of $] i, j[$, that is, if $S=] i, j[$ (resp. $S=\varnothing$ ). We say that $\Sigma(i, j, S)$ is mixed if the corresponding arc crosses the horizontal axis, that is, if $\varnothing \neq S \subsetneq] i, j[$.

Figures 3 and 4 illustrate the braid fans $\mathcal{F}_{n}$ and their shards $\boldsymbol{\Sigma}_{n}$ when $n=3$ and $n=4$ respectively. As the 3 -dimensional fan $\mathcal{F}_{4}$ is difficult to visualize (as in Figure 1 (middle)), we use another classical representation in Figure 4 (left): we intersect $\mathcal{F}_{4}$ with a unit sphere and we stereographically project the resulting arrangement of great circles from the pole 4321 to the plane. Each circle then corresponds to a hyperplane $\boldsymbol{x}_{i}=\boldsymbol{x}_{j}$ with $i<j$, separating a disk where $\boldsymbol{x}_{i}<\boldsymbol{x}_{j}$ from an unbounded region where $\boldsymbol{x}_{i}>\boldsymbol{x}_{j}$. In both Figures 3 and 4 , the left picture shows the braid fan $\mathcal{F}_{n}$ (where chambers are labeled with blue permutations of $[n]$ and rays are labeled with red proper subsets of $[n]$ ), the
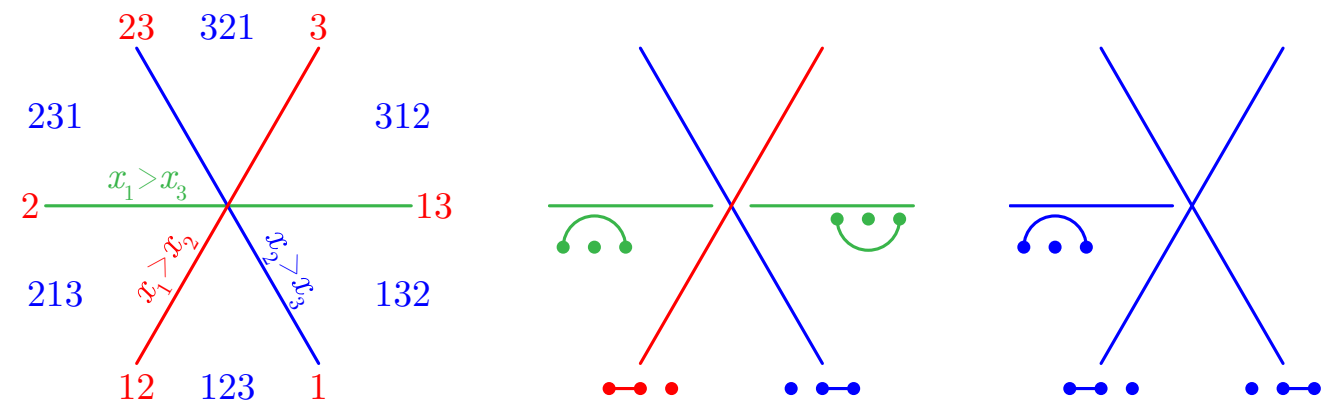

Figure 3: The braid fan $\mathcal{F}_{3}$ (left), the corresponding shards (middle), and the quotient fan of the sylvester congruence $\equiv_{\text {sylv }}$ (right). [PS19, Fig. 3] 

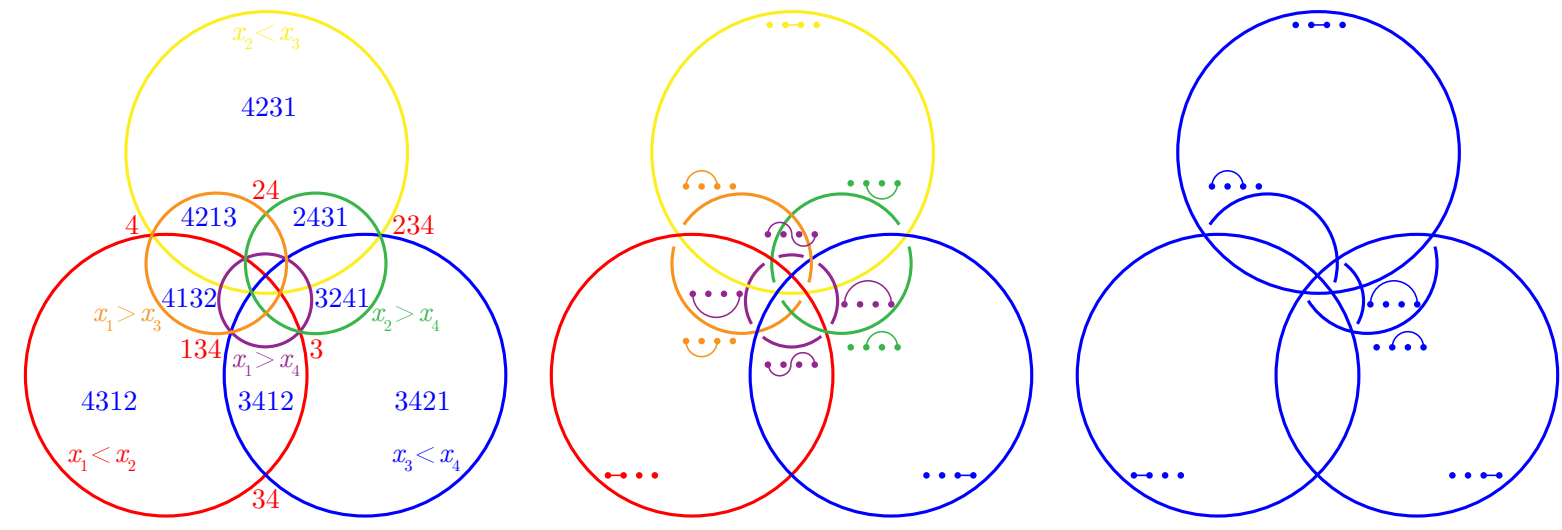

Figure 4: A stereographic projection of the braid fan $\mathcal{F}_{4}$ (left) from the pole 4321, the corresponding shards (middle), and the quotient fan of the sylvester congruence $\equiv_{\text {sylv }}$ (right). [PS19, Fig. 4]

middle picture shows the shards $\boldsymbol{\Sigma}_{n}$ (labeled by arcs), and the right picture represents the quotient fan $\mathcal{F}_{\text {sylv }}$ by the sylvester congruence.

It turns out that the shards are precisely the pieces of the hyperplanes of $\mathcal{H}_{n}$ needed to delimit the cones of the quotient fan $\mathcal{F}_{\equiv}$.

Theorem 9 ([Rea16a, Sect. 10.5]). For any lattice congruence $\equiv$ of the weak order on $\mathfrak{S}_{n}$, there is a subset of shards $\boldsymbol{\Sigma}_{\equiv} \subseteq \boldsymbol{\Sigma}_{n}$ such that the interiors of the chambers of the fan $\mathcal{F}_{\equiv}$ are precisely the connected components of $\mathbb{H} \backslash \bigcup \Sigma_{\equiv}$.

Finally, we can describe the set of lattice congruences of the weak order on $\mathfrak{S}_{n}$ using the following poset on the shards $\Sigma_{n}$. A shard $\Sigma(i, j, S)$ is said to force a shard $\Sigma(h, k, T)$ if $h \leqslant i<j \leqslant k$ and $S=T \cap] i, j[$. We denote this relation by $\Sigma(i, j, S) \succ \Sigma(h, k, T)$. In terms of the corresponding arcs, the $\operatorname{arc} \alpha$ of $\Sigma(i, j, S)$ is a subarc of the $\operatorname{arc} \beta$ of $\Sigma(h, k, T)$, meaning that the endpoints of $\alpha$ are in between the endpoints of $\beta$, and the arc $\alpha$ agrees with the $\operatorname{arc} \beta$ between $i$ and $j$. We call shard poset the poset $\left(\boldsymbol{\Sigma}_{n}, \prec\right)$ of all shards ordered by forcing. The forcing relation and the shard poset on $\Sigma_{4}$ are illustrated on Figure 5 .
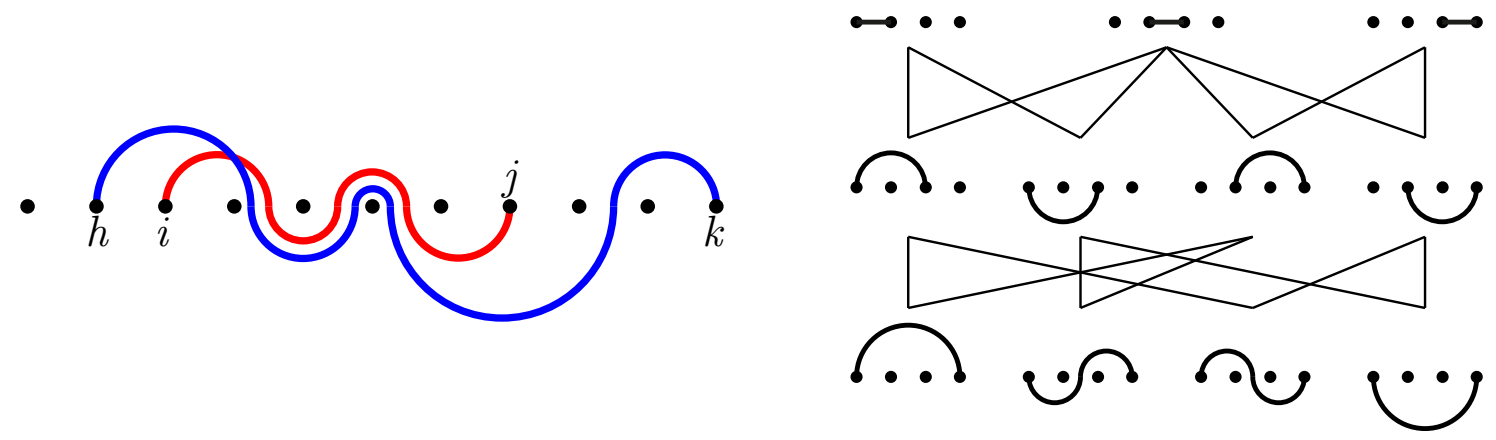

Figure 5: The forcing relation on shards (left) and the shard poset for $n=4$ (right). The red shard $\Sigma(i, j, S)$ forces the blue shard $\Sigma(h, k, T)$ since $h \leqslant i<j \leqslant k$ and $S=T \cap] i, j[$. [PS19, Fig. 5] 
Theorem 10 ([Rea16a, Sect. 10.5]). The map $\equiv \mapsto \boldsymbol{\Sigma}_{\equiv}$ is a bijection between the lattice congruences of the weak order on $\mathfrak{S}_{n}$ and the upper ideals of the shard poset $\left(\boldsymbol{\Sigma}_{n}, \prec\right)$.

Example 11. For the sylvester congruence $\equiv_{\text {sylv }}$, the corresponding shard ideal is the ideal $\boldsymbol{\Sigma}_{\mathrm{sylv}}=\{\Sigma(i, j] i,, j[) \mid 1 \leqslant i<j \leqslant n\}$ of all up shards, i.e. those whose corresponding arcs pass above all dots in between their endpoints. Figures 3 and 4 (right) represent the quotient fans $\mathcal{F}_{\equiv_{\text {sylv }}}$ corresponding to the sylvester congruences $\equiv_{\text {sylv }}$ on $\mathfrak{S}_{3}$ and $\mathfrak{S}_{4}$ respectively. It is obtained

- either by gluing the chambers $C(\sigma)$ of the permutations $\sigma$ in the same sylvester class,

- or by cutting the space with the shards of $\boldsymbol{\Sigma}_{\equiv_{\text {sylv }}}=\{\Sigma(i, j] i,, j[) \mid 1 \leqslant i<j \leqslant 4\}$.

To conclude these recollections on lattice congruences of the weak order, let us recall that the quotient fan $\mathcal{F}_{\equiv}$ is essential if and only if the identity permutation is alone in its $\equiv$-congruence class, or equivalently if $\boldsymbol{\Sigma}_{\equiv}$ contains all basic shards $\Sigma(i, i+1, \varnothing)$ for $i \in[n-1]$ (this follows e.g. from [Rea04, Thm. 6.9]). We say that such a congruence is essential. If $\boldsymbol{\Sigma}_{\equiv}$ does not contain the shard $\Sigma(i, i+1, \varnothing)$, then the quotient $\mathfrak{S}_{n} / \equiv$ is isomorphic to the Cartesian product of the quotients $\mathfrak{S}_{i} / \equiv^{\prime}$ and $\mathfrak{S}_{n-i} / \equiv^{\prime \prime}$ where $\equiv^{\prime}$ and $\equiv^{\prime \prime}$ are the restrictions of $\equiv$ to $[1, i]$ and $[i+1, n]$ respectively. Any lattice congruence can thus be understood from its essential restrictions and we therefore focus on essential congruences.

\subsection{Deformed permutahedra and removahedra}

This section discusses the type cone of the braid fan $\mathcal{F}_{n}$. As they belong to the deformation cone of the permutahedron, we call the resulting polytopes deformed permutahedra. We also discuss a subfamily of specific deformed permutahedra called removahedra as they are obtained by deleting facets (instead of moving them).

\subsubsection{Linear dependences in the braid fan}

We start with classical considerations on the geometry of the braid fan. Remember that we have chosen a representative vector $\boldsymbol{r}(I):=|I| \mathbf{1}-n \mathbf{1}_{I}$ for the ray corresponding to each proper subset $\varnothing \neq I \subsetneq[n]$ (where $\mathbf{1}:=\sum_{i \in[n]} \boldsymbol{e}_{i}$ and $\mathbf{1}_{I}:=\sum_{i \in I} \boldsymbol{e}_{i}$ ). We also set $\boldsymbol{r}(\varnothing)=\boldsymbol{r}([n])=0$ by convention.

Lemma 12. For any two proper subsets $\varnothing \neq I, J \subsetneq[n]$, the representative vectors satisfy the linear dependence $\boldsymbol{r}(I)+\boldsymbol{r}(J)=\boldsymbol{r}(I \cap J)+\boldsymbol{r}(I \cup J)$.

Lemma 13. Let $\sigma, \tau$ be two adjacent permutations. Let $\varnothing \neq I \subsetneq[n]($ resp. $\varnothing \neq J \subsetneq[n])$ be the two proper subsets such that $\boldsymbol{r}(I)$ (resp. $\boldsymbol{r}(J)$ ) is the ray of $C(\sigma)$ not in $C(\tau)$ (resp. of $C(\tau)$ not in $C(\sigma)$ ). Then the linear dependence among the rays of the cones $C(\sigma)$ and $C(\tau)$ is $\boldsymbol{r}(I)+\boldsymbol{r}(J)=\boldsymbol{r}(I \cap J)+\boldsymbol{r}(I \cup J)$. 
For example, the linear dependence among the rays in the adjacent cones $C(123)$ and $C(213)$ of $\mathcal{F}_{3}$ is $\boldsymbol{r}(\{1\})+\boldsymbol{r}(\{2\})=\boldsymbol{r}(\{12\})$, while the linear dependence among the rays in the adjacent cones $C(123)$ and $C(132)$ of $\mathcal{F}_{3}$ is $\boldsymbol{r}(\{1,2\})+\boldsymbol{r}(\{1,3\})=\boldsymbol{r}(\{1\})$. See Figure 3 (left). The first non-degenerate linear dependencies (i.e. where $I \cap J \neq \varnothing$ and $I \cup J \neq[n])$ arise in $\mathcal{F}_{4}$ : for instance, the linear dependence among the rays in the adjacent cones $C(4132)$ and $C(4312)$ of $\mathcal{F}_{4}$ is $\boldsymbol{r}(\{3,4\})+\boldsymbol{r}(\{1,4\})=\boldsymbol{r}(\{4\})+\boldsymbol{r}(\{1,3,4\})$. See Figure 4 (left).

\subsubsection{Deformed permutahedra}

We now consider the type cone of the braid fan, or in other words the deformation cone of the permutahedron, studied in details in [Pos09, PRW08]. The following classical statement is a consequence of Proposition 2 and Lemma 13. We naturally identify a vector $\boldsymbol{h}$ with coordinates indexed by the rays of the braid fan $\mathcal{F}_{n}$ with a height function $h: 2^{[n]} \rightarrow \mathbb{R}_{\geqslant 0}$ with $h(\varnothing)=h([n])=0$.

Proposition 14. The type cone $\overline{\mathbb{T} C}\left(\mathcal{F}_{n}\right)$ of the braid fan $\mathcal{F}_{n}$ (or deformation cone of the permutahedron Perm $_{n}$ ) is (isomorphic to) the set of functions $h: 2^{[n]} \rightarrow \mathbb{R}_{\geqslant 0}$ satisfying $h(\varnothing)=h([n])=0$ and the submodular inequalities $h(I)+h(J) \geqslant h(I \cap J)+h(I \cup J)$ for any $I, J \subseteq[n]$. The facets of $\overline{\mathbb{T} C}\left(\mathcal{F}_{n}\right)$ correspond to those submodular inequalities where $|I \backslash J|=|J \backslash I|=1$.

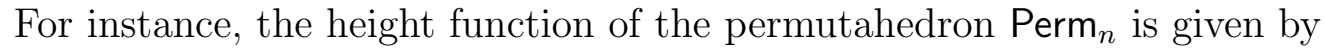

$$
h_{\circ}(I)=\max _{\sigma \in \mathfrak{S}_{n}}\langle\boldsymbol{r}(I) \mid \sigma\rangle=|I| n(n+1) / 2-n|I|(|I|+1) / 2=n|I|(n-|I|) / 2 .
$$

It is clearly submodular since $h_{\circ}(I)+h_{\circ}(J)-h_{\circ}(I \cap J)-h_{\circ}(I \cup J)=2 n|I \backslash J||J \backslash I| \geqslant 0$.

A deformed permutahedron is a polytope whose normal fan coarsens the braid fan $\mathcal{F}_{n}$. It can be written as $\operatorname{Defo}_{h}:=\{\boldsymbol{x} \in \mathbf{H} \mid\langle\boldsymbol{r}(I) \mid \boldsymbol{x}\rangle \leqslant h(I)$ for all $\varnothing \neq I \subsetneq[n]\}$ for some submodular function $h: 2^{[n]} \rightarrow \mathbb{R}$. We prefer the term deformed permutahedron to the term generalized permutahedron used by A. Postnikov in [Pos09, PRW08] (in particular because it also generalizes to other Coxeter groups [ACEP20]). Observe in particular that any quotientope $P_{\equiv}$ of Theorem 7 is a deformed permutahedron since the quotient fan $\mathcal{F}_{\equiv}$ coarsens the braid fan $\mathcal{F}_{n}$ by definition.

\subsubsection{Removahedra}

A removahedron is a deformed permutahedron obtained by deleting inequalities in the facet description of the permutahedron Perm ${ }_{n}$. In other words, it can be written as $\operatorname{Remo}_{\mathcal{I}}:=\left\{\boldsymbol{x} \in \mathbf{H} \mid\langle\boldsymbol{r}(I) \mid \boldsymbol{x}\rangle \leqslant h_{\circ}(I)\right.$ for all $\left.I \in \mathcal{I}\right\}$ for a subset $\mathcal{I}$ of proper subsets of $[n]$. Examples of removahedra include the permutahedron Perm ${ }_{n}$ itself (remove no inequality), the associahedron $\mathrm{Asso}_{n}$ (remove the inequalities that do not correspond to intervals), the graph associahedron Asso $_{G}$ [CD06, Dev09] if and only if the graph $G$ is chordful [Pil17], and the permutreehedra [PP18] described below.

We say that a fan $\mathcal{G}$ with rays $\{\boldsymbol{r}(I) \mid I \in \mathcal{I}\}$ is removahedral if $\mathcal{G}$ is the normal fan of the removahedron $\operatorname{Remo}_{\mathcal{I}}$. We say that a lattice congruence $\equiv$ of the weak order is 
removahedral if its quotient fan $\mathcal{F}_{\equiv}$ is. The following example shows that some lattice congruence are not removahedral.

Example 15. Consider the congruence $\equiv$ of $\mathfrak{S}_{4}$ with shard ideal $\boldsymbol{\Sigma}_{\equiv}=\boldsymbol{\Sigma}_{4} \backslash\{\Sigma(1,4,\{2\})\}$. Since the only removed shard contains no ray in its interior, the rays of the quotient fan $\mathcal{F}_{\equiv}$ are all rays of the braid fan $\mathcal{F}_{\equiv}$, so that the corresponding removahedron is the permutahedron Perm $_{4}$ which does not realize $\mathcal{F}_{\equiv}$.

\subsection{Permutrees}

We now recall the permutrees of [PP18] that generalize the binary trees and will be especially important in this paper. The presentation and pictures are borrowed from [PP18].

\subsubsection{Combinatorics of permutrees}

In an oriented tree $T$, we call parents (resp. children) of a node $j$ the outgoing (resp. incoming) neighbors of $j$, and ancestor (resp. descendant) subtrees of $j$ the connected components of the parents (resp. children) of $j$ in $T \backslash\{j\}$. A permutree is an oriented tree $T$ with nodes $[n]$, such that

- any node has either one or two parents and either one or two children,

- if node $j$ has two parents (resp. children), then all nodes in its left ancestor (resp. descendant) subtree are smaller than $j$ while all nodes in its right ancestor (resp. descendant) subtree are larger than $j$.

Figure 6 provides four examples of permutrees. We use the following conventions:

- All edges are oriented bottom-up and the nodes appear from left to right in natural order.

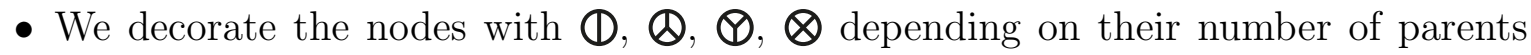
and children. The sequence of these symbols, from left to right, is the decoration $\delta$ of $T$. We let $\delta^{-}:=\left\{j \in[n] \mid \delta_{j}=\boldsymbol{Q}\right.$ or $\left.\boldsymbol{\otimes}\right\}$ and $\delta^{+}:=\left\{j \in[n] \mid \delta_{j}=\boldsymbol{\bigotimes}\right.$ or $\left.\boldsymbol{\otimes}\right\}$.

- We draw a vertical red wall below (resp. above) the nodes of $\delta^{-}$(resp. of $\delta^{+}$) to mark the separation between the left and right descendant (resp. ancestor) subtrees.

As illustrated in Figure 6, $\delta$-permutrees extend and interpolate between various combinatorial families, including permutations when $\delta=\mathbb{D}^{n}$, binary trees when $\delta=\mathbb{Q}^{n}$, and binary sequences when $\delta=\boldsymbol{\bigotimes}^{n}$. In fact, permutrees arose by pushing further the combinatorics of Cambrian trees developed in [CP17] to provide combinatorial models to the type $A$ Cambrian lattices [Rea06].

An edge cut in a permutree $T$ is the partition $(I \| J)$ of the nodes of $T$ into the set $I$ of nodes in the source set and the set $J=[n] \backslash I$ of nodes in the target set of an oriented edge of $T$. Edge cuts play an important role in the geometry of the permutree fan defined below. 

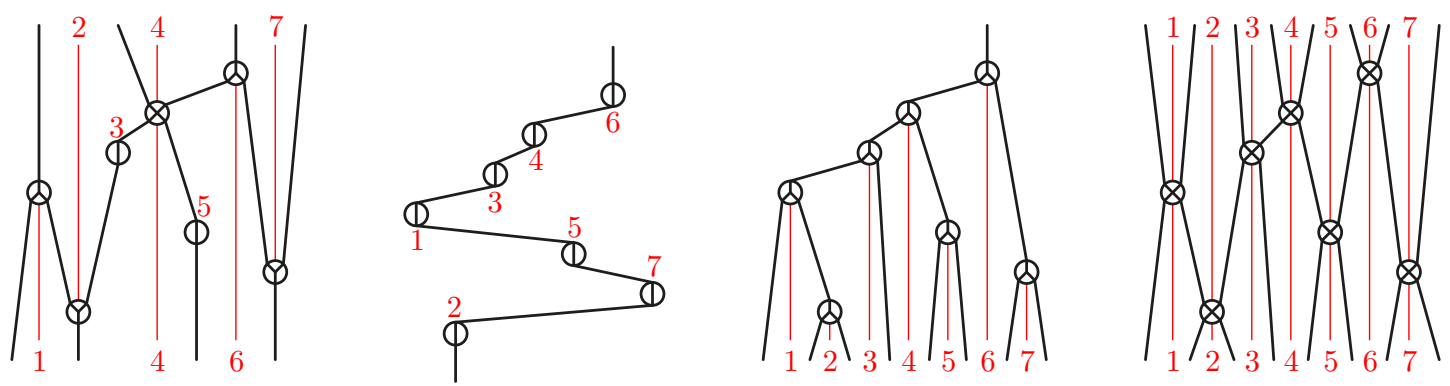

Figure 6: Four examples of permutrees. While the first is generic, the last three use decorations corresponding to permutations, binary trees, and binary sequences. [PP18, Fig. $2 \& 3]$

As with the classical rotation operation on binary trees, there is a local operation on $\delta$ permutrees which only exchanges the orientation of an edge and rearranges the endpoints of two other edges. Namely, consider an edge $i \rightarrow j$ in a $\delta$-permutree $T$. Let $D$ denote the only (resp. the right) descendant subtree of node $i$ if $i \notin \delta^{-}$(resp. if $i \in \delta^{-}$) and let $U$ denote the only (resp. the left) ancestor subtree of node $j$ if $j \notin \delta^{+}$(resp. if $j \in \delta^{+}$). Let $S$ be the oriented tree obtained from $T$ by just reversing the orientation of $i \rightarrow j$ and attaching the subtree $U$ to $i$ and the subtree $D$ to $j$. The transformation from $T$ to $S$ is the rotation of the edge $i \rightarrow j$.

Proposition 16 ([PP18]). The rotation of an edge $i \rightarrow j$ in a $\delta$-permutree $T$ produces a $\delta$-permutree $S$. Moreover, $S$ is the unique $\delta$-permutree with the same edge cuts as $T$, except the cut defined by $i \rightarrow j$.

Define the increasing rotation graph as the directed graph whose vertices are the $\delta$ permutrees and whose arcs are increasing rotations $T \rightarrow S$, i.e. where the edge $i \rightarrow j$ in $T$ is reversed to the edge $i \leftarrow j$ in $S$ for $i<j$. See Figure 7 .

Proposition 17 ([PP18]). The transitive closure of the increasing rotation graph on $\delta$-permutrees is a lattice, called $\delta$-permutree lattice.

The $\delta$-permutree lattice specializes to the weak order when $\delta=\mathbb{D}^{n}$, the Tamari lattice when $\delta=\mathbb{Q}^{n}$, the Cambrian lattices [Rea06] when $\delta \in\{\otimes, \otimes\}^{n}$ and the boolean lattice when $\delta=\bigotimes^{n}$.

In fact, all permutree lattices are lattice quotients of the weak order, exactly as the Tamari lattice is the quotient of the weak order by the sylvester congruence. The $\delta$-permutree congruence $\equiv_{\delta}$ is the equivalence relation on $\mathfrak{S}_{n}$ defined equivalently as:

- the relation whose equivalence classes are given by the sets of linear extensions of the $\delta$-permutrees,

- the relation whose equivalence classes are the fibers of the $\delta$-permutree insertion, similar to the binary tree insertion and presented in detail in [PP18], 

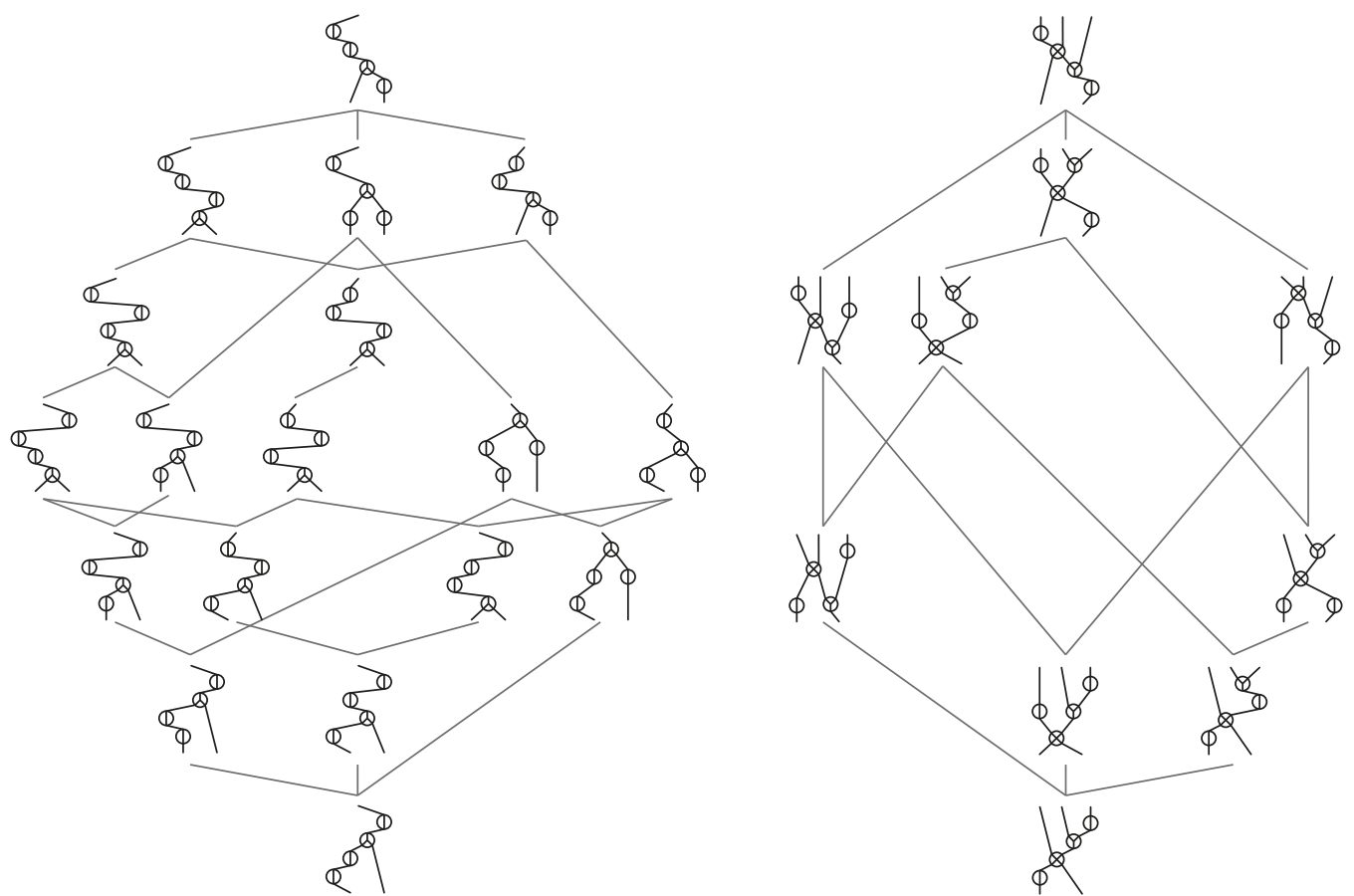

Figure 7: The $\delta$-permutree lattices for $\delta=$ ФDQФ (left) and $\delta=(1 \otimes \otimes(1)$ (right). [PP18, Fig. 11]

- the transitive closure of the rewriting rules $U i k V j W \equiv_{\delta} U k i V j W$ when $j \in \delta^{-}$and $U j V i k W \equiv_{\delta} U j V k i W$ when $j \in \delta^{+}$, for some letters $i<j<k$ and words $U, V, W$ on $[n]$,

- the congruence of the weak order with arc ideal

$$
\boldsymbol{\Sigma}_{\delta}:=\left\{\Sigma(i, j, S) \mid 1 \leqslant i<j \leqslant n, \delta^{-} \cap\right] i, j[\subseteq S \subseteq] i, j\left[\backslash \delta^{+}\right\} .
$$

In other words, the corresponding arcs do not pass below any $k \in \delta^{-}$nor above any $k \in \delta^{+}$.

This extends the trivial congruence when $\delta=\mathbb{Q}^{n}$, the sylvester congruence [HNT05] when $\delta=\mathbb{Q}^{n}$, the Cambrian congruences [Rea04, Rea06, CP17] when $\delta \in\{\boldsymbol{Q}, \mathbb{Q}\}^{n}$, and the hypoplactic congruence [KT97, Nov00] when $\delta=\boldsymbol{\otimes}^{n}$. The corresponding shard ideals are represented in Figure 8.

Proposition 18 ([PP18]). The $\delta$-permutree congruence $\equiv_{\delta}$ is a lattice congruence of the weak order and the quotient $\mathfrak{S}_{n} / \equiv_{\delta}$ is (isomorphic to) the $\delta$-permutree lattice.

Note that the description of the shard ideal $\Sigma_{\delta}$ above gives the following characterization of permutree congruences.

Proposition 19. A lattice congruence $\equiv$ is a permutree congruence if and only if all generators of the lower ideal $\Sigma_{n} \backslash \Sigma_{\equiv}$ are of length 2 , i.e. of the form $\Sigma(i-1, i+1, \varnothing)$ or $\Sigma(i-1, i+1,\{i\})$. 

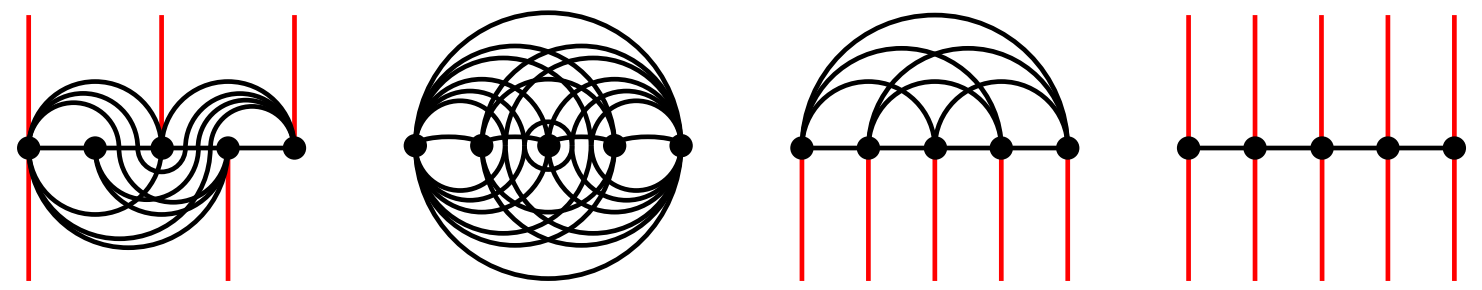

Figure 8: Four examples of permutree shard ideals. While the first is generic, the last three use decorations corresponding to permutations, binary trees, and binary sequences. [Pil19, Fig. 3]

We conclude these recollections on combinatorics of permutrees with a natural order on all permutree congruences. For two decorations $\delta, \delta^{\prime} \in\{\mathbb{D}, \mathbb{Q}, \boldsymbol{Q}, \mathbb{\otimes}\}^{n}$, we say that $\delta$ refines $\delta^{\prime}$ and we write $\delta \preccurlyeq \delta^{\prime}$ if $\delta_{i} \preccurlyeq \delta_{i}^{\prime}$ for all $i \in[n]$ for the order $\mathbb{(} \preccurlyeq\{\otimes, \bigotimes\} \preccurlyeq \bigotimes$. In this case, the $\delta$-permutree congruence refines the $\delta^{\prime}$-permutree congruence: $\sigma \equiv_{\delta} \tau$ implies $\sigma \equiv_{\delta^{\prime}} \tau$ for any two permutations $\sigma, \tau \in \mathfrak{S}_{n}$.

\subsubsection{Geometry of permutrees}

We finally recall the geometric constructions of [PP18] realizing the $\delta$-permutree lattice.

The $\delta$-permutree fan $\mathcal{F}_{\delta}$ is the quotient fan of the $\delta$-permutree congruence. It has

- a chamber $C(T)$ for each $\delta$-permutree $T$, which can be defined either as the union of the chambers $C(\sigma)$ for all linear extensions $\sigma$ of $T$, or by the inequalities $\boldsymbol{x}_{i} \leqslant \boldsymbol{x}_{j}$ for all edges $i \rightarrow j$ in $T$, or by the generators $|I| \mathbf{1}_{J}-|J| \mathbf{1}_{I}$ for all edge cuts $(I \| J)$ of $T$,

- a ray $C(I)$ for each proper subset $\varnothing \neq I \subsetneq[n]$ such that for all $a<b<c$, if $a, c \in I$ then $b \notin \delta^{-} \backslash I$, and if $a, c \notin I$ then $b \notin \delta^{+} \cap I$. We denote by $\mathcal{I}_{\delta}$ the collection of these proper subsets. See Proposition 32.

Two examples of $\delta$-permutree fans are represented in Figure 9 . The $\delta$-permutree fan $\mathcal{F}_{\delta}$ specializes to the braid fan when $\delta=\mathbb{D}^{n}$, the (type A) Cambrian fans of N. Reading and D. Speyer [RS09] when $\delta \in\{\otimes, \bigotimes\}^{n}$, the fan defined by the hyperplane arrangement $\boldsymbol{x}_{i}=\boldsymbol{x}_{i+1}$ for each $i \in[n-1]$ when $\delta=\boldsymbol{\otimes}^{n}$, and the fan defined by the hyperplane arrangement $\boldsymbol{x}_{i}=\boldsymbol{x}_{j}$ for each $1 \leqslant i<j \leqslant n$ such that $\delta_{k}=$ (1) for all $i<k<j$ when $\delta \in\{\mathbb{D}, \otimes\}^{n}$. Decoration refinements translate to permutree fan refinements: if $\delta \preccurlyeq \delta^{\prime}$, then the $\delta$-permutree fan $\mathcal{F}_{\delta}$ refines the $\delta^{\prime}$-permutree fan $\mathcal{F}_{\delta^{\prime}}$.

The $\delta$-permutreehedron $\mathrm{PT}_{\delta}$ is the polytope defined equivalently as:

- the convex hull of the points $\sum_{j \in[n]}(1+d(T, j)+\underline{\ell}(T, j) \underline{r}(T, j)-\bar{\ell}(T, j) \bar{r}(T, j)) \boldsymbol{e}_{j}$ for all $\delta$-permutrees $T$, where $d(T, j), \underline{\ell}(T, j), \underline{r}(T, j), \bar{\ell}(T, j), \bar{r}(T, j)$ respectively denote the numbers of nodes in the descendant, left descendant, right descendant, left ancestor, right ancestor subtrees of $j$ in $T$,

- the intersection of the hyperplane $\mathbf{H}:=\left\{\boldsymbol{x} \in \mathbb{R}^{n} \mid \sum_{i \in[n]} \boldsymbol{x}_{i}=\left(\begin{array}{c}n+1 \\ 2\end{array}\right)\right\}$ with the halfspaces $\left\{\boldsymbol{x} \in \mathbb{R}^{n} \mid \sum_{i \in I} \boldsymbol{x}_{i} \geqslant\left(\begin{array}{c}|I|+2 \\ 2\end{array}\right)\right\}$ for all subsets $I$ in $\mathcal{I}_{\delta}$. 

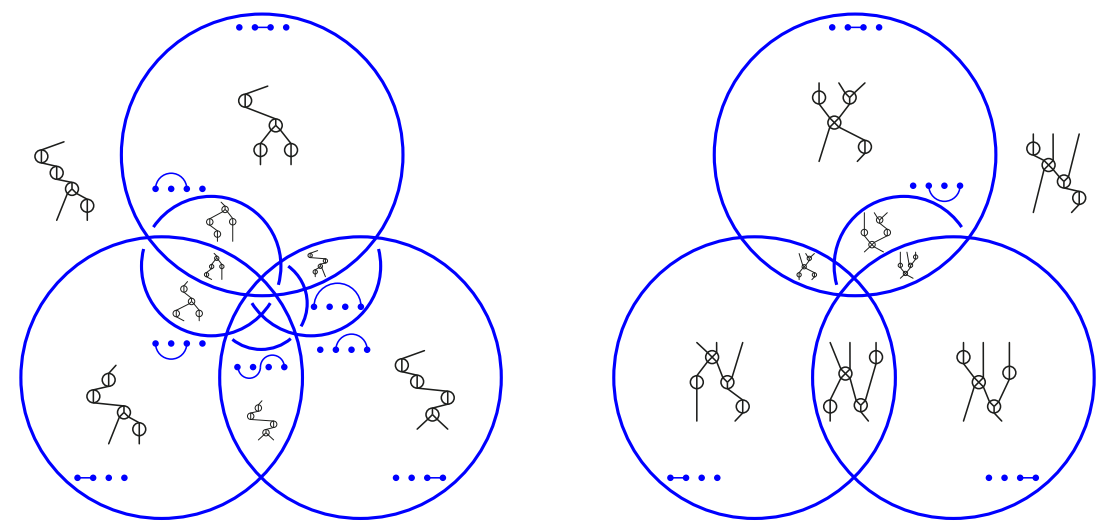

Figure 9: The permutree Fans $\mathcal{F}_{\Phi \Phi \otimes \Phi}$ (left) and $\mathcal{F}_{\Phi \otimes \bigotimes Ф}$ (right). Each shard is labeled with its coresponding arc, and some chambers are labeled with their corresponding permutrees.
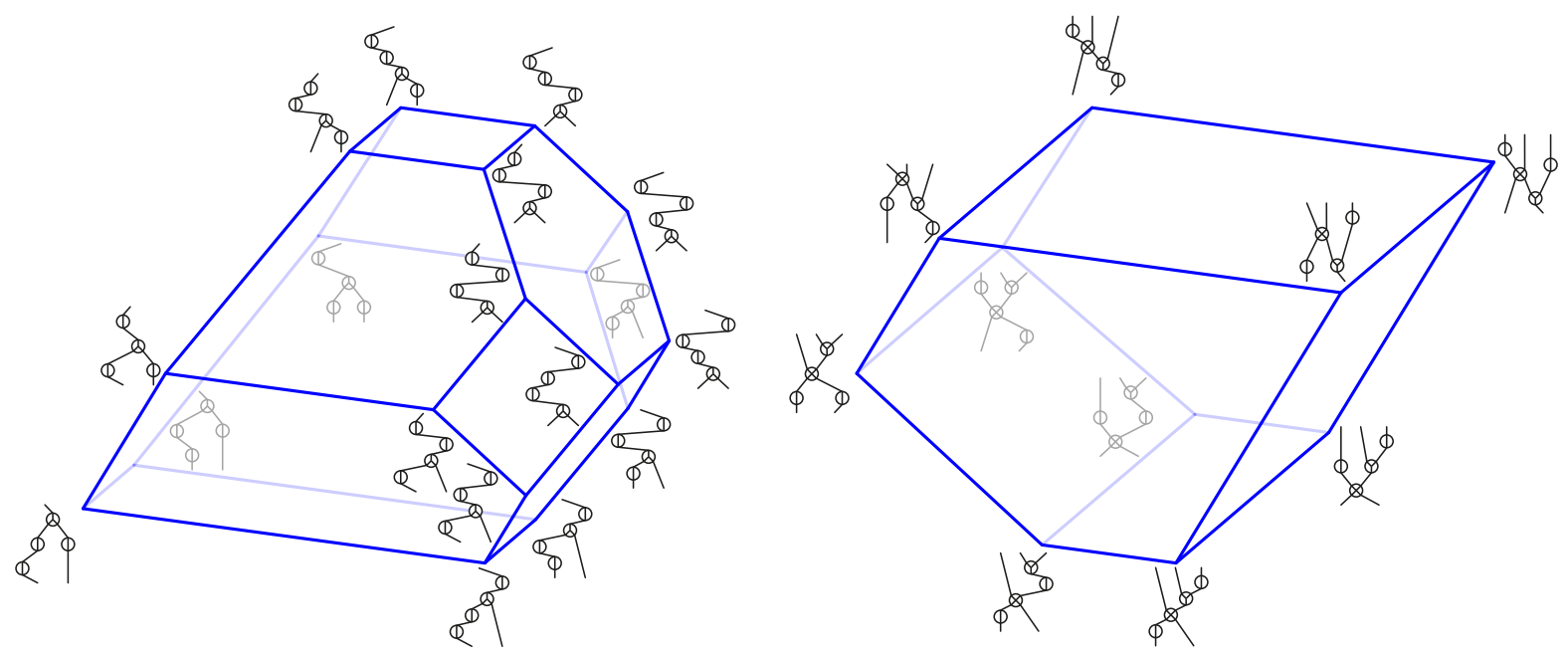

Figure 10: The permutreehedra $P T_{\Phi Ф \otimes Ф}$ (left) and $\mathrm{PT} \Phi \otimes \otimes \oplus$ (right). [PP18, Fig. 15]

Two examples of $\delta$-permutreehedra are represented in Figure 10. The $\delta$-permutreehedron $\mathrm{PT}_{\delta}$ specializes to the permutahedron Perm $n$ when $\delta=\mathbb{D}^{n}$, J.-L. Loday's associahedron Asso $_{n}$ [SS93, Lod04] when $\delta=\mathbb{Q}^{n}$, C. Hohlweg and C. Lange's associahe$\operatorname{dra} \mathrm{Asso}_{\delta}[\mathrm{HL} 07, \mathrm{LP} 18]$ when $\delta \in\{\boldsymbol{\bigotimes}, \boldsymbol{Q}\}^{n}$, the parallelepiped with directions $\boldsymbol{e}_{i}-\boldsymbol{e}_{i+1}$ for each $i \in[n-1]$ when $\delta=\boldsymbol{\otimes}^{n}$, the graphical zonotope Zono $\delta$ generated by the vectors $\boldsymbol{e}_{i}-\boldsymbol{e}_{j}$ for each $1 \leqslant i<j \leqslant n$ such that $\delta_{k}=\mathbb{( 1 )}$ for all $i<k<j$ when $\delta \in\{\mathbb{(}, \otimes\}^{n}$.

Theorem 20 ([PP18]). The $\delta$-permutree fan $\mathcal{F}_{\delta}$ is the normal fan of the $\delta$-permutreehedron $\mathrm{PT}_{\delta}$.

Note that decoration refinements translate to permutreehedra inclusions: if $\delta \preccurlyeq \delta^{\prime}$, then the permutreehedron $\mathrm{PT}_{\delta^{\prime}}$ is obtained by deleting inequalities in the facet description of the permutreehedron $\mathrm{PT}_{\delta^{\prime}}$, so that $\mathrm{PT}_{\delta} \subseteq \mathrm{PT}_{\delta^{\prime}}$ as illustrated in Figure 11. In particular, 


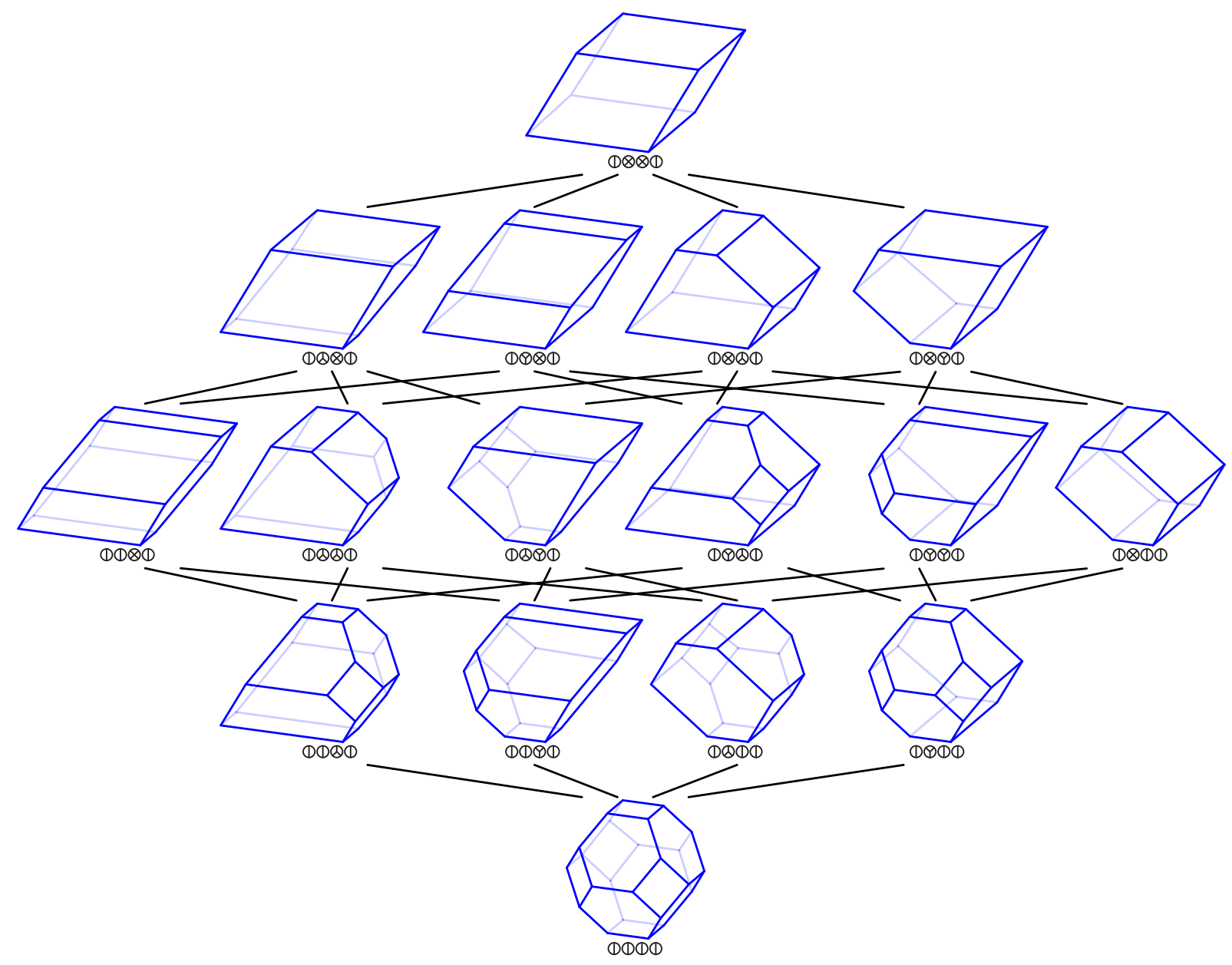

Figure 11: The $\delta$-permutreehedra, for all decorations $\delta \in \mathbb{(} \cdot\{\mathbb{(}, \mathbb{Q}, \mathbb{Q}, \mathbb{Q}\}^{2} \cdot(\mathbb{D}$. [PP18, Fig. 16]

all facet-defining inequalities of the permutreehedron $\mathrm{PT}_{\delta}$ are facet-defining inequalities of the permutahedron Perm ${ }_{n}$, which can be rephrased as follows.

Corollary 21. All permutree congruences are removahedral.

\section{Removahedral congruences}

The main goal of this section is to show Theorem 1. An important step of the proof is the description of the rays of the quotient fans, provided in Section 3.1. While elementary, we are not aware that a characterization of these rays appeared in the literature. We then proceed to prove Theorem 1 (i) in Section 3.2 and Theorem 1 (ii) in Section 3.3. 


\subsection{Rays of the quotient fan}

Fix a ray $\boldsymbol{r}(I)$ of the braid fan $\mathcal{F}_{n}$ corresponding to a proper subset $\varnothing \neq I \subsetneq[n]$. The shards of $\boldsymbol{\Sigma}_{n}$ containing $\boldsymbol{r}(I)$ were characterized by a simple combinatorial criterion in [PS19, Lem. 5]. Here, we need to characterize which shards of $\boldsymbol{\Sigma}_{n}$ contain $\boldsymbol{r}(I)$ in their relative interior. We associate with $I$ the set $\boldsymbol{\Sigma}_{I}$ of $n-2$ shards containing the $|I|-1$ down shards joining two consecutive elements of $I$ and the $n-|I|-1$ up shards joining two consecutive elements of $[n] \backslash I$, that is

$$
\Sigma_{I}:=\{\Sigma(i, j, \varnothing) \mid i, j \in I \text { and }] i, j[\cap I=\varnothing\} \cup\{\Sigma(i, j,] i, j[) \mid i, j \notin I \text { and }] i, j[\subseteq I\} .
$$

Lemma 22. The $n-2$ shards of $\boldsymbol{\Sigma}_{I}$ are the only shards containing $\boldsymbol{r}(I)$ in their relative interior.

Proof. Recall that

- the ray $\boldsymbol{r}(I)$ is given by $|I| \mathbf{1}-n \mathbf{1}_{I}$, thus $\boldsymbol{x}_{i}<\boldsymbol{x}_{j}$ for $i \in I$ and $j \notin I$,

- the relative interior of $\Sigma(i, j, S)$ is given by $\boldsymbol{x}_{h}<\boldsymbol{x}_{i}=\boldsymbol{x}_{j}<\boldsymbol{x}_{k}$ for all $h \in S$ and $k \notin] i, j[\backslash S$.

Hence, $\boldsymbol{r}(I)$ satisfies these inequalities if and only if either $i, j \in I, S=\varnothing$ and $] i, j[\cap I=\varnothing$, or $i, j \notin I, S=] i, j[$, and $] i, j[\subseteq I$. Therefore, $\boldsymbol{r}(I)$ is contained in the relative interior of $n-2$ shards.

Note that this implies that a mixed shard (i.e. of the form $\Sigma(i, j, S)$ with $S \notin\{\varnothing] i,, j[\}$, or said differently whose arc crosses the horizontal axis) contains no ray of the braid fan in its relative interior. See for example the shards $\Sigma(1,4,\{2\})$ and $\Sigma(1,4,\{3\})$ in Figure 4.

From Lemma 22, we derive the following description of the rays of the quotient fan $\mathcal{F}_{\equiv}$. As an illustration, this description is specialized to permutree fans in Proposition 32.

Lemma 23. The ray $\boldsymbol{r}(I)$ is a ray of the quotient fan $\mathcal{F}_{\equiv}$ if and only if $\boldsymbol{\Sigma}_{\equiv}$ contains $\boldsymbol{\Sigma}_{I}$.

Proof. If $\Sigma_{\equiv}$ contains $\boldsymbol{\Sigma}_{I}$, then the quotient fan $\mathcal{F}_{\equiv}$ contains $n-2$ shards which intersect along $\boldsymbol{r}(I)$, so that $\boldsymbol{r}(I)$ is a ray of $\mathcal{F}_{\equiv}$. The converse can be derived from [Rea05, Prop. 5.10] or [Rea11, Prop. 7.7]. Let us just provide a sketchy argument. Let $\mathfrak{S}_{I}$ be the interval of permutations $\sigma$ of $[n]$ such that cone $C(\sigma)$ contains $\boldsymbol{r}(I)$ (or equivalently $\sigma([|I|])=I)$. Let $\equiv_{I}$ denote the subcongruence of $\equiv$ induced by $\mathfrak{S}_{I}$. The basic shards of $\equiv_{I}$ are the shards of $\boldsymbol{\Sigma}_{I}$. Since $\boldsymbol{\Sigma}_{\equiv}$ does not contain $\boldsymbol{\Sigma}_{I}$, the congruence $\equiv_{I}$ is not essential, so that $\boldsymbol{r}(I)$ is not a ray of $\mathcal{F}_{\equiv}$.

\subsection{Removahedral congruences}

We are now ready to prove the following statement, which is a more precise reformulation of Theorem 1 (i).

Theorem 24. The only essential removahedral congruences are the permutree congruences. 
Note that we focus here on essential congruences. However, as already mentioned, nonessential congruences can be understood from their essential restrictions. For arbitrary congruences, Theorem 24 says that the essential restrictions of removahedral congruences are permutree congruences.

We learned from Corollary 21 that permutree congruences are essential removahedral congruences, and we want to prove the opposite direction. We thus assume by contradiction that there exists an essential removahedral congruence $\equiv$ which is not a permutree congruence.

Lemma 25. The generating set $\boldsymbol{\Pi}_{\equiv}$ of the lower ideal $\boldsymbol{\Sigma}_{n} \backslash \boldsymbol{\Sigma}_{\equiv}$ contains at least one shard of the form $(i, j, \varnothing)$ or $(i, j] i,, j[)$ for $i \leqslant j-3$.

Proof. Since $\equiv$ is essential, $\boldsymbol{\Pi}_{\equiv}$ contains no shard of length 1 . Since $\equiv$ is not a permutree congruence, $\boldsymbol{\Pi}_{\equiv}$ must contain a shard of length distinct from 2 by Proposition 19. Decompose the set of shards $\boldsymbol{\Pi}_{\equiv}$ into the subset $\boldsymbol{\Pi}_{\equiv}^{=2}$ of shards of length 2 and the subset $\boldsymbol{\Pi}_{\equiv}^{>2}$ of shards of length greater than 3 . Let $\equiv_{\delta}$ denote the permutree congruence defined by $\boldsymbol{\Pi}_{\equiv_{\delta}}=\boldsymbol{\Pi}_{\equiv}^{=2}$. If $\boldsymbol{\Pi}_{\equiv}^{>2}$ contains only mixed shards, then $\equiv$ and $\equiv_{\delta}$ contain the same up and down shards, since mixed shards only force mixed shards. This implies by Lemma 23 that the quotient fans $\mathcal{F}_{\equiv}$ and $\mathcal{F}_{\delta}$ have the same rays. The corresponding removahedron is thus the permutreehedron $\mathrm{PT}_{\delta}$ which does not realize the quotient fan $\mathcal{F}_{\equiv}$ so that $\equiv$ is not removahedral. The statement follows.

We assume now that there are $i \leqslant j-3$ such that the shard $\Sigma(i, j] i,, j[)$ is one of the generators of the lower ideal $\Sigma_{n} \backslash \boldsymbol{\Sigma}_{\equiv}$. The proof for the $\operatorname{shard} \Sigma(i, j, \varnothing)$ is symmetric. We consider the following five subsets of $[n]$ :

$$
I=] i+1, j[, \quad J=] i, j-1[, \quad K=[1, j[, \quad L=] i, n] \quad \text { and } \quad M=] i+1, j-1[.
$$

Note that $M$ might be empty, in which case it will not appear in all the computations below. Before showing that these subsets provide a contradiction, let us consider a minimal example.

Example 26. Consider the lattice congruence $\equiv$ for $n=4$ whose only deleted shard is $\Sigma(1,4,\{2,3\})$. Consider the subsets

$$
I=\{3\}, \quad J=\{2\}, \quad K=\{1,2,3\} \quad \text { and } \quad L=\{2,3,4\} .
$$

(Note that $M=\varnothing$ is irrelevant here.) Then the cones $C:=\mathbb{R}_{\geqslant 0}\{\boldsymbol{r}(I), \boldsymbol{r}(K), \boldsymbol{r}(L)\}$ and $D:=\mathbb{R}_{\geqslant 0}\{\boldsymbol{r}(J), \boldsymbol{r}(K), \boldsymbol{r}(L)\}$ are two adjacent chambers of the quotient fan $\mathcal{F}_{\equiv}$, whose corresponding linear dependence is $\boldsymbol{r}(I)+\boldsymbol{r}(J)=\boldsymbol{r}(K)+\boldsymbol{r}(L)$. However, the height function $h_{\circ}(I)=4|I|(4-|I|) / 2$ of the permutahedron Perm 4 satisfies $h_{\circ}(I)+h_{\circ}(J)=$ $h_{\circ}(K)+h_{\circ}(L)$, thus violating the wall-crossing inequality $h_{\circ}(I)+h_{\circ}(J)>h_{\circ}(K)+h_{\circ}(L)$. Therefore, the lattice congruence $\equiv$ is not removahedral.

We now come back to the general situation. We need the following three observations. 
Lemma 27. The rays $\boldsymbol{r}(I), \boldsymbol{r}(J), \boldsymbol{r}(K), \boldsymbol{r}(L)$, and $\boldsymbol{r}(M)$ are all rays of the quotient fan $\mathcal{F}_{\equiv}$.

Proof. By Lemma 22, the only non-basic shards containing these rays in their interior are

- $\Sigma(i+1, j] i+1,, j[)$ for $I$,

- $\Sigma(i, j-1] i,, j-1[)$ for $J$,

- $\Sigma(i+1, j-1] i+1,, j-1[)$ for $M$.

Since all these shards are in $\boldsymbol{\Sigma}_{\equiv}$ by minimality of $\Sigma(i, j] i,, j[)$, the result follows by Lemma 23.

Lemma 28. The quotient fan $\mathcal{F}_{\equiv}$ contains two chambers $C$ and $D$ which are adjacent along the hyperplane $\left\{\boldsymbol{x} \in \mathbb{R}^{n} \mid \boldsymbol{x}_{i+1}=\boldsymbol{x}_{j-1}\right\}$, such that $\boldsymbol{r}(I) \in C$ while $\boldsymbol{r}(J) \in D$ and $\{\boldsymbol{r}(K), \boldsymbol{r}(L), \boldsymbol{r}(M)\} \subset C \cap D$.

Proof. Observe that for two proper subsets $\varnothing \neq I, J \subsetneq[n]$, the rays $\boldsymbol{r}(I)$ and $\boldsymbol{r}(J)$ are separated by the hyperplane of equation $\boldsymbol{x}_{i}=\boldsymbol{x}_{j}$ if and only if $i \in I \backslash J$ and $j \in J \backslash I$ or vice versa. Therefore, $I \subseteq J$ implies that $\boldsymbol{r}(I)$ and $\boldsymbol{r}(J)$ belong to a common chamber of the braid arrangement $\mathcal{F}_{n}$ (in fact to the chamber $C(\sigma)$ for any permutation $\sigma$ such that $\sigma([|I|])=I$ and $\sigma([|J|])=J)$. Since $M \subseteq I, J \subseteq K, L$, the only rays separated by an hyperplane are:

- $\boldsymbol{r}(I)$ and $\boldsymbol{r}(J)$ which are separated by the hyperplane of equation $\boldsymbol{x}_{i+1}=\boldsymbol{x}_{j-1}$,

- $\boldsymbol{r}(K)$ and $\boldsymbol{r}(L)$ which are separated by all hyperplanes of equation $\boldsymbol{x}_{k}=\boldsymbol{x}_{\ell}$ for $k \leqslant i$ and $\ell \geqslant j$. However, $\boldsymbol{r}(K)$ and $\boldsymbol{r}(L)$ belong to the same chamber of the quotient fan $\mathcal{F}_{\equiv}$ since we remove all shards $\Sigma(k, \ell] k,, \ell[)$ for $k \leqslant i$ and $\ell \geqslant j$.

For completeness, let us provide a less intuitive but more formal alternative argument for this statement. Consider a sequence of permutations starting with the permutation $\sigma:=[i+2, \ldots, j-2, j-1, i+1, i, \ldots, 1, j, \ldots, n]$ and ending with the permutation $\tau:=[i+2, \ldots \ldots, j-2, j-1, i+1, j, \ldots, n, i, \ldots, 1]$, and obtained by transposing at each step two values $k \leqslant i$ and $\ell \geqslant j$ at consecutive positions. In other words, all the permutations in the sequence start by $[i+2, \ldots, j-2, j-1, i+1]$ and end with a shuffle of $[i, \ldots, 1]$ with $[j, \ldots, n]$. At each step, the interval $] k, \ell[$ between the two transposed values always appears before the position of the transposition. Therefore, the chambers corresponding to the two permutations before and after the transposition are separated by the shard $\Sigma(k, \ell] k,, \ell[)$, which does not belong to $\Sigma_{\equiv}$ since it is forced by $\Sigma(i, j] i,, j[)$. It follows that the cones of all these permutations, and in particular those of $\sigma$ and $\tau$, belong to the same chamber $C$ of the quotient fan $\mathcal{F}_{\equiv}$. This chamber $C$ contains the rays $\boldsymbol{r}(I), \boldsymbol{r}(K), \boldsymbol{r}(L)$ and $\boldsymbol{r}(M)$ since the subsets $I$ and $M$ (resp. $K$, resp. $L$ ) are initial intervals of all permutations in the sequence (resp. of $\sigma$, resp. of $\tau$ ). We prove similarly that $\boldsymbol{r}(J), \boldsymbol{r}(K), \boldsymbol{r}(L)$ and $\boldsymbol{r}(M)$ belong to a chamber $D$ by considering a sequence of permutations starting with $[i+2, \ldots, j-2, i+1, j-1, i, \ldots, 1, j, \ldots, n]$ and ending with $[i+2, \ldots, j-2, i+1, j-1, i, \ldots, 1, j, \ldots, n]$. 
Lemma 29. Let $h_{\circ}(I)=n|I|(n-|I|) / 2$ be the height function of the classical permutahedron Perm $_{n}$. Then

- $\boldsymbol{r}(I)+\boldsymbol{r}(J)=\boldsymbol{r}(K)+\boldsymbol{r}(L)+\boldsymbol{r}(M)$,

- $h_{\circ}(I)+h_{\circ}(J)-h_{\circ}(K)-h_{\circ}(L)-h_{\circ}(M)=n i(j-n)+n(1-i) \leqslant 0$.

Proof. Immediate computations from the cardinalities

$$
|I|=|J|=j-i-2, \quad|K|=j-1, \quad|L|=n-i \quad \text { and } \quad|M|=j-i-3 .
$$

Observe that the inequality $h_{\circ}(I)+h_{\circ}(J) \leqslant h_{\circ}(K)+h_{\circ}(L)+h_{\circ}(M)$ is an equality if and only if $i=1$ and $j=n$, as was the case in Example 26. We have now all ingredients to conclude the proof of Theorem 24 .

Proof of Theorem 24. Combining Lemmas 27 to 29, we obtain that the height function $h_{\circ}(I)=n|I|(n-|I|) / 2$ of the permutahedron violates the wall-crossing inequality $h_{\circ}(I)+h_{\circ}(J)>h_{\circ}(K)+h_{\circ}(L)+h_{\circ}(M)$ of Proposition 4 corresponding to the linear dependence $\boldsymbol{r}(I)+\boldsymbol{r}(J)=\boldsymbol{r}(K)+\boldsymbol{r}(L)+\boldsymbol{r}(M)$ between the rays of the adjacent chambers $C$ and $D$ of the quotient fan $\mathcal{F}_{\equiv}$, so that $\equiv$ is not removahedral.

\subsection{Permutree congruences are strongly removahedral}

We now prove that the permutree congruences are removahedral in a stronger sense. Namely, we show that we obtain polytopes realizing the permutree fans by deleting inequalities in the facet description of any polytope realizing the braid fan, not only the

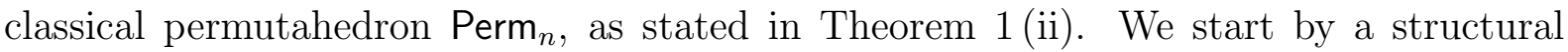
observation on the exchanges in permutree fans.

Proposition 30. Consider two adjacent chambers $\mathbb{R}_{\geqslant 0} \boldsymbol{R}$ and $\mathbb{R}_{\geqslant 0} \boldsymbol{S}$ of the $\delta$-permutree fan $\mathcal{F}_{\delta}$ with $\boldsymbol{R} \backslash \boldsymbol{S}=\{\boldsymbol{r}(I)\}$ and $\boldsymbol{S} \backslash \boldsymbol{R}=\{\boldsymbol{r}(J)\}$. Then the rays $\boldsymbol{r}(I \cap J)$ and $\boldsymbol{r}(I \cup J)$ are also rays of the $\delta$-permutree fan $\mathcal{F}_{\delta}$ and belong to $\boldsymbol{R} \cap \boldsymbol{S}$. Therefore, all wall-crossing inequalities of the $\delta$-permutree fan $\mathcal{F}_{\delta}$ are of the form

$$
h(I)+h(J)>h(I \cap J)+h(I \cup J),
$$

with the usual convention that $h(\varnothing)=h([n])=0$.

Proof. Consider the two $\delta$-permutrees $T$ and $S$ whose chambers are $C(T)=\mathbb{R}_{\geqslant 0} \boldsymbol{R}$ and $C(S)=\mathbb{R}_{\geqslant 0} \boldsymbol{S}$. Let $i \rightarrow j$ denote the edge of $T$ that is rotated to the edge $j \rightarrow i$ in $S$. Up to swapping the roles of $I$ and $J$, we can assume that $i<j$. We denote by $U$,

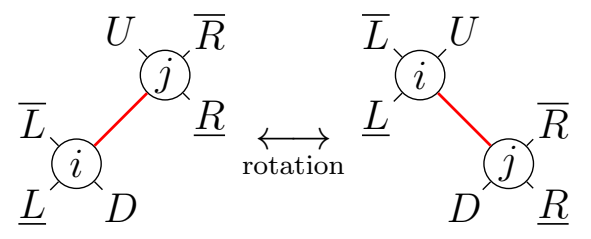
$D, \underline{L}, \bar{L}, \underline{R}, \bar{R}$ the subtrees of $T$ and $S$ as illustrated on the figure on the right. Note that some of the subtrees $\underline{L}, \bar{L}, \underline{R}, \bar{R}$ might not exist if $\delta_{i} \neq \otimes$ or $\delta_{j} \neq \otimes$. We then just assume that they are empty. Since the rays of the cone $C(T)$ are given by $|I| \mathbf{1}_{J}-|J| \mathbf{1}_{I}$ 
for all edge cuts of $(I \| J)$ of $T$, and the unique edge cut that differs from $T$ to $S$ is that corresponding to the edge $i-j$ by Proposition 16, we obtain that

$$
I=\{i\} \cup D \cup \underline{L} \cup \bar{L} \quad \text { and } \quad J=\{j\} \cup D \cup \underline{R} \cup \bar{R}
$$

and therefore that

$$
I \cap J=D \quad \text { and } \quad I \cup J=\{i, j\} \cup D \cup \underline{L} \cup \bar{L} \cup \underline{R} \cup \bar{R}=[n] \backslash U .
$$

But $(D \|[n] \backslash D)$ and $([n] \backslash U \| U)$ are edge cuts in the $\delta$-permutrees $T$ and $S$, so that the rays $\boldsymbol{r}(I \cap J)$ and $\boldsymbol{r}(I \cup J)$ are rays of the $\delta$-permutree fan $\mathcal{F}_{\delta}$ and belong to $\boldsymbol{R} \cap \boldsymbol{S}$. We therefore obtain that the unique (up to rescaling) linear dependence among the rays of $\boldsymbol{R} \cup \boldsymbol{S}$ is $\boldsymbol{r}(I)+\boldsymbol{r}(J)=\boldsymbol{r}(I \cap J)+\boldsymbol{r}(I \cup J)$. The corresponding wall-crossing inequality is thus given by $h(I)+h(J)>h(I \cap J)+h(I \cup J)$.

Corollary 31. For any strictly submodular function $h$ (i.e. a function $h: 2^{[n]} \rightarrow \mathbb{R}_{\geqslant 0}$ such that $h(\varnothing)=h([n])=0$ and $h(I)+h(J) \geqslant h(I \cap J)+h(I \cup J)$ for all $I, J \subseteq[n])$, and any decoration $\delta \in\{\mathbb{(}, \otimes, \otimes, \otimes\}^{n}$, the $\delta$-permutree fan $\mathcal{F}_{\delta}$ is the normal fan of the polytope

$$
\mathrm{PT}_{\delta}^{h}:=\left\{\boldsymbol{x} \in \mathbf{H} \mid\langle\boldsymbol{r}(I) \mid x\rangle \leqslant h(I) \text { for all } I \in \mathcal{I}_{\delta}\right\},
$$

where $\mathcal{I}_{\delta}=\left\{\varnothing \neq I \subsetneq[n] \mid \boldsymbol{r}(I)\right.$ is a ray of $\left.\mathcal{F}_{\delta}\right\}$ (see Proposition 32 for a characterization). In other words, we obtain a polytope realizing the $\delta$-permutree fan $\mathcal{F}_{\delta}$ by deleting inequalities in the facet description of any polytope realizing the braid arrangement $\mathcal{F}_{n}$.

\section{Type cones of permutree fans}

In this section, we provide a complete facet description of the type cone $\overline{\mathbb{T} C}\left(\mathcal{F}_{\delta}\right)$ of the $\delta$-permutree fan $\mathcal{F}_{\delta}$. We first describe the rays of $\mathcal{F}_{\delta}$ in Section 4.1 , then the pairs of exchangeable rays of $\mathcal{F}_{\delta}$ in Section 4.2 , and finally the facets of the type cone $\overline{\mathbb{T}} \mathbb{C}\left(\mathcal{F}_{\delta}\right)$ in Section 4.3. We conclude by a description of kinematic permutreehedra for the permutree fans $\mathcal{F}_{\delta}$ whose type cone is simplicial in Section 4.4.

\subsection{Rays of permutree fans}

Specializing Lemma 23 to the $\delta$-permutree congruence $\equiv_{\delta}$, we obtain the following description of the rays of the $\delta$-permutree fan $\mathcal{F}_{\delta}$ announced in Section 2.4.2.

Proposition 32. A ray $\boldsymbol{r}(I)$ is a ray in the $\delta$-permutree fan $\mathcal{F}_{\delta}$ if and only if for all $a<b<c$, if $a, c \in I$ then $b \notin \delta^{-} \backslash I$, and if $a, c \notin I$ then $b \notin \delta^{+} \cap I$.

Example 33. For the decorations of Figures 7, 9 and 10, the rays of $\mathcal{F}_{\Phi \oplus \otimes \Phi}$ correspond to the subsets $1,2,3,4,12,13,23,34,123,134,234$ while the rays of $\mathcal{F}_{\mathbb{\Phi} \otimes \otimes \oplus}$ correspond to the subsets $1,4,12,34,123,124,234$.

Example 34. Specializing Proposition 32, we recover the following classical descriptions: 
- when $\delta=\mathbb{D}^{n}$, the rays of the braid fan $\mathcal{F}_{\mathbb{\Phi}^{n}}$ are all proper subsets $\varnothing \neq I \subsetneq[n]$,

- when $\delta=\bigotimes^{n}$, the rays of $\mathcal{F}_{\bigotimes^{n}}$ are all proper intervals $[i, j]$ of $[n]$, (equivalently, one can think of the interval $[i, j]$ as corresponding to the internal diagonal $(i-1, j+1)$ of a polygon with vertices labeled $0, \ldots, n+1)$,

- when $\delta=\boldsymbol{Q}^{n}$, the rays of $\mathcal{F}_{\mathbb{\Phi}^{n}}$ are all proper initial intervals $[1, i]$ or final intervals $[i, n]$.

Proof of Proposition 32. We have $a, c \in I$ and $b \in \delta^{-} \backslash I$ if and only if the shard $\Sigma(i, j, \varnothing)$ is in $\Sigma_{I}$ but not in $\Sigma_{\delta}$, where $i:=\max ([a, b[\cap I)$ and $j:=\min (] b, c] \cap I)$. Similarly, $a, c \notin I$ and $b \in \delta^{+} \cap I$ if and only if the shard $\Sigma(i, j] i,, j[)$ is in $\Sigma_{I}$ but not in $\boldsymbol{\Sigma}_{\delta}$, where $i:=\max ([a, b[\backslash I)$ and $j:=\min (] b, c] \backslash I)$. The statement thus follows from Lemma 23.

An alternative argument would be to observe directly that these conditions are necessary and sufficient to allow the construction of a $\delta$-permutree with an edge whose cut is $(I \|[n] \backslash I)$.

Corollary 35. The number $\rho(\delta)$ of rays of the $\delta$-permutree fan $\mathcal{F}_{\delta}$ is

$$
\rho(\delta)=n-1+\sum_{\substack{1 \leqslant i<j \leqslant n \\ \forall i<k<j, \delta_{k} \neq \otimes}} 2^{\left|\left\{i<k<j \mid \delta_{k}=\Phi\right\}\right|} .
$$

Example 36. For the decorations of Figures 7, 9 and 10, we obtain $\rho($ (D囚Ф) $=11$

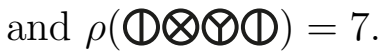

Example 37. Specializing the formula of Corollary 35, we recover the following classical numbers:

- when $\delta=\mathbb{\Phi}^{n}$, the braid fan $\mathcal{F}_{\mathbb{D}^{n}}$ has $2^{n}-2$ rays,

- when $\delta=\bigotimes^{n}$, the fan $\mathcal{F}_{\bigotimes^{n}}$ has $\left(\begin{array}{c}n+1 \\ 2\end{array}\right)-1$ rays (equalling the number of internal diagonals of the $(n+2)$-gon),

- when $\delta=\boldsymbol{\otimes}^{n}$, the fan $\mathcal{F}_{\otimes^{n}}$ has $2 n-2$ rays.

Proof of Corollary 35. To choose a ray $\boldsymbol{r}(I)$ of $\mathcal{F}_{\delta}$, we proceed as follows:

- We choose the last position $i$ (resp. first position $j$ ) such that $1, \ldots, i$ (resp. $j, \ldots, n$ ) all belong to $I$ or all belong to $[n] \backslash I$. Note that $1 \leqslant i<j \leqslant n$.

- For any $i<k<j$, since $|\{i, i+1\} \cap I|=1$ and $|\{j-1, j\} \cap I|=1$, the characterization of Proposition 32 imposes that $k \in I$ if $k \in \delta^{-}$, and $k \notin I$ if $k \in \delta^{+}$. This is impossible if $\delta_{k}=\otimes$ (explaining the condition over the sum), and leaves two choices if $\delta_{k}=$ (1) (explaining the power of 2).

- If $i+1<j$, then the presence of $i+1$ (resp. $j-1$ ) in $I$ requires the absence of $i$ (resp. $j$ ) in $I$ and vice versa, so there is no choice left. 
- If $i+1=j$, we have counted only one ray while both subsets $[1, i]$ and $[j, n]$ indeed correspond to rays of $\mathcal{F}_{\delta}$.

Corollary 38. If $\delta \in\{\otimes, \otimes, \otimes\}^{n}$, we have

$$
\rho(\delta)=n-1+\left|\left\{1 \leqslant i<j \leqslant n \mid \forall i<k<j, \delta_{k} \neq \otimes\right\}\right| .
$$

\subsection{Exchangeable rays of permutree fans}

An immediate corollary of Proposition 30 is that the linear dependence between the rays of two adjacent chambers $C:=\mathbb{R}_{\geqslant 0} \boldsymbol{R}$ and $D:=\mathbb{R}_{\geqslant 0} \boldsymbol{S}$ of $\mathcal{F}_{\delta}$ with $\boldsymbol{R} \backslash \boldsymbol{S}=\{\boldsymbol{r}\}$ and $\boldsymbol{S} \backslash \boldsymbol{R}=\{\boldsymbol{s}\}$ only depend on the rays $\boldsymbol{r}$ and $\boldsymbol{s}$, not on the chambers $C$ and $D$. This property is called unique exchange relation property in [PPPP19] and allows to describe the type cone by inequalities associated with exchangeable rays rather than with walls.

We therefore proceed in identifying the pairs of exchangeable rays of $\mathcal{F}_{\delta}$. We consider two subsets $I, J \in \mathcal{I}_{\delta}$, i.e. proper subsets $\varnothing \neq I, J \subsetneq[n]$ such that $\boldsymbol{r}(I)$ and $\boldsymbol{r}(J)$ are rays of the $\delta$-permutree fan $\mathcal{F}_{\delta}$, as characterized in Proposition 32.

Proposition 39. The rays $\boldsymbol{r}(I)$ and $\boldsymbol{r}(J)$ are exchangeable in the $\delta$-permutree fan $\mathcal{F}_{\delta}$ if and only if, up to swapping the roles of $I$ and $J$,

(i) $i:=\max (I \backslash J)<\min (J \backslash I)=: j$,

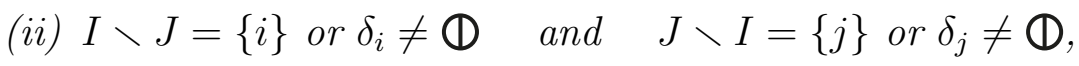

(iii) $] i, j\left[\cap \delta^{-} \subseteq I \cap J \quad\right.$ and $\left.\quad\right] i, j\left[\cap \delta^{+} \cap I \cap J=\varnothing\right.$.

Example 40. For the decorations of Figures 7, 9 and 10, the pairs of exchangeable rays of $\mathcal{F}_{\Phi Ф \otimes \Phi}$ correspond to the pairs of subsets $\{1,2\},\{1,3\},\{1,34\},\{12,13\},\{12,134\}$, $\{12,23\},\{12,234\},\{123,134\},\{123,234\},\{123,4\},\{13,23\},\{13,34\},\{13,4\},\{134,234\}$, $\{2,3\},\{2,34\},\{23,34\},\{23,4\},\{3,4\}$, while the pairs of exchangeable rays of $\mathcal{F}_{\Phi \otimes \otimes}$ correspond to the pairs of subsets $\{1,234\},\{12,34\},\{12,4\},\{123,124\},\{123,4\},\{124,34\}$.

Example 41. Specializing Proposition 39, we recover that the pairs of exchangeable rays in $\mathcal{F}_{\delta}$ correspond to the pairs of proper subsets $\{I, J\}$ where

- when $\delta=\mathbb{D}^{n}$, we have $I=K \cup\{i\}$ and $J=K \cup\{j\}$ for $1 \leqslant i<j \leqslant n$ and $K \subseteq[n] \backslash\{i, j\}$,

- when $\delta=\bigotimes^{n}$, we have $I=[h, j[$ and $J=] i, k]$ for some $1 \leqslant h \leqslant i<j \leqslant k \leqslant n$, (equivalently, the internal diagonals $(h-1, j)$ and $(i, k+1)$ of the $(n+2)$-gon intersect),

- when $\delta=\boldsymbol{\bigotimes}^{n}$, we have $I=[1, i]$ and $\left.\left.J=\right] i, n\right]$ for some $1 \leqslant i<n$. 
Proof of Proposition 39. We first prove that the conditions of Proposition 39 are necessary for the rays $\boldsymbol{r}(I)$ and $\boldsymbol{r}(J)$ to be exchangeable in the $\delta$-permutree fan $\mathcal{F}_{\delta}$. We keep the notations of the proof of Proposition 30. Remember that we had $I=\{i\} \cup D \cup \underline{L} \cup \bar{L}$ and $J=\{j\} \cup D \cup \underline{R} \cup \bar{R}$. Since $\underline{L} \cup \bar{L}<i<j<\underline{R} \cup \bar{L}$, we obtain that $i=\max (I \backslash J)$ and $j=\min (J \backslash I)$ indeed satisfy (i). Moreover, $I \backslash J=\{i\} \cup \underline{L} \cup \bar{L}$ is restricted to $\{i\}$ if $\delta_{i}=\mathbb{( 1 )}$ (because the subtrees $\underline{L}$ and $\bar{L}$ must then be empty), and similarly $J \backslash I=\{j\} \cup \underline{R} \cup \bar{R}$ is restricted to $\{j\}$ if $\delta_{j}=\mathbb{D}$, wich shows (ii). Finally, if there is $i<k<j$ such that $k \in \delta^{-} \backslash(I \cap J)$ (resp. $k \in \delta^{+} \cap(I \cap J)$ ), then the edge $i-j$ crosses the red wall below $k$ (resp. above $k$ ), which shows (iii).

Assume now that $I$ and $J$ satisfy the conditions of Propositions 32 and 39 . We construct two $\delta$-permutrees $T$ and $S$, connected by the rotation of the edge $i-j$ whose edge cut in $T$ is $I$ and in $S$ is $J$. For this, we first pick an arbitrary permutree, that we denote by $D$ (resp. $U$, resp. $L$, resp. $R$ ), for the restriction of the decoration $\delta$ to the subset $I \cap J$ (resp. $[n] \backslash(I \cup J)$, resp. $I \backslash J \backslash\{i\}$, resp. $J \backslash I \backslash\{j\})$. We then construct an oriented tree $T$ on $[n]$ starting with an edge $i \rightarrow j$ and placing

- $D$ as the only (resp. the right) descendant subtree of $i$ if $i \notin \delta^{-}$(resp. if $i \in \delta^{-}$),

- $U$ as the only (resp. the left) ancestor subtree of $j$ if $j \notin \delta^{+}$(resp. if $j \in \delta^{+}$),

- $L$ as the left descendant (resp. ancestor) subtree of $i$ if $i \in \delta^{-}$(resp. if $i \notin \delta^{-}$),

- $R$ as the right descendant (resp. ancestor) subtree of $j$ if $j \in \delta^{-}$(resp. if $j \notin \delta^{-}$).

Note that there is only one way to place these subtrees. For instance, to place $D$, we connect the leftmost upper blossom of $D$ to the only (resp. the right) lower blossom of $i$ if $i \notin \delta^{-}$(resp. if $i \in \delta^{-}$).

We claim that the conditions of Propositions 32 and 39 ensure that $T$ is a $\delta$-permutree. Observe first that it indeed forms a tree since the permutree $L$ (resp. $R$ ) is empty if $\delta_{i}=$ (1) (resp. $\delta_{j}=(1)$ by Proposition 39 (ii). Hence, we just need to show that no edge of $T$ crosses a red wall below a node $i \in \delta^{-}$or above a node $i \in \delta^{+}$. Since all nodes of $L$ (resp. $R$ ) are smaller than $i$ (resp. $j$ ) by Proposition 39 (i), and there is no red wall above (resp. below) the nodes of $D$ (resp. $U$ ) between $i$ and $j$ by Proposition 39 (iii), the edge $i \rightarrow j$ crosses no red wall. Consider now an edge $\ell-\ell^{\prime}$ of $L \cup\{i\}$ with $\ell<\ell^{\prime}$. It cannot cross a red wall emanating from a node $r$ of $R \cup\{j\}$ since $\ell^{\prime} \leqslant i<j \leqslant r$, nor from a node $u$ of $U$ since otherwise we would have $\ell<u<\ell^{\prime}$ with $\ell, \ell^{\prime} \in I$ and $u \in \delta^{-} \backslash I$ contradicting Proposition 32, nor from a node $d$ of $D$ since otherwise we would have $\ell<d<\ell^{\prime}$ with $\ell, \ell^{\prime} \notin J$ and $d \in \delta^{+} \cap J$ contradicting Proposition 32. We prove similarly that no edge in $D \cup\{i\}$, nor in $U \cup\{j\}$, nor in $R \cup\{j\}$ crosses a red wall. This closes the proof that $T$ is a $\delta$-permutree.

Finally, denote by $S$ the $\delta$-permutree obtained by the rotation of the edge $i \rightarrow j$ in $T$. Observe that the construction is done so that the edge $i \rightarrow j$ in $T$ has cut $(I \|[n] \backslash I)$ while the edge $j \rightarrow i$ in $S$ has cut $(J \|[n] \backslash J)$. It follows that the rays $\boldsymbol{r}(I)$ and $\boldsymbol{r}(J)$ are exchangeable in the adjacent chambers $C(T)$ and $C(S)$ of the $\delta$-permutree fan $\mathcal{F}_{\delta}$. 
Corollary 42. The number $\chi(\delta)$ of pairs of exchangeable rays in the $\delta$-permutree fan $\mathcal{F}_{\delta}$ is

$$
\chi(\delta)=\sum_{\substack{1 \leqslant i<j \leqslant n \\ \forall i<k<j, \delta_{k} \neq \otimes}} \Omega\left(\delta_{1} \ldots \delta_{i-1}\right)^{\delta_{i} \neq \otimes} \cdot 2^{\left|\left\{i<k<j \mid \delta_{k}=\Phi\right\}\right|} \cdot \Omega\left(\delta_{n} \ldots \delta_{j+1}\right)^{\delta_{j} \neq \otimes},
$$

where $\Omega\left(\delta_{1} \ldots \delta_{k}\right)$ is defined inductively from $\Omega(\varepsilon)=1$ by

$$
\Omega\left(\delta_{1} \ldots \delta_{k}\right)= \begin{cases}2 \cdot \Omega\left(\delta_{1} \ldots \delta_{k-1}\right) & \text { if } \delta_{k}=\mathbb{D}, \\ 1+\Omega\left(\delta_{1} \ldots \delta_{k-1}\right) & \text { if } \delta_{k} \in\{\boldsymbol{Q}, \boldsymbol{\bigotimes}\} \\ 2 & \text { if } \delta_{k}=\boldsymbol{\otimes} .\end{cases}
$$

Example 43. For the decorations of Figures 7, 9 and 10, we obtain $\chi($ (DQ円) $=19$ and $\chi(1 \otimes \otimes \emptyset)=6$.

Example 44. Specializing the formula of Corollary 42, we recover the following classical numbers:

- when $\delta=\mathbb{D}^{n}$, the braid fan $\mathcal{F}_{\mathbb{\Phi}^{n}}$ has $2^{n-2}\left(\begin{array}{l}n \\ 2\end{array}\right)$ pairs of exchangeable rays,

- when $\delta=\bigotimes^{n}$, the fan $\mathcal{F}_{\bigotimes^{n}}$ has $\left(\begin{array}{c}n+2 \\ 4\end{array}\right)$ pairs of exchangeable rays (equalling the number of quadruples of vertices of the $(n+2)$-gon),

- when $\delta=\mathbb{\bigotimes}^{n}$, the fan $\mathcal{F}_{\mathbb{\otimes}^{n}}$ has $n-1$ pairs of exchangeable rays.

Proof of Corollary 42. A pair of exchangeable rays in the $\delta$-permutree fan $\mathcal{F}_{\delta}$ is a pair of proper subsets $\varnothing \neq I, J \subsetneq[n]$ satisfying the conditions of Propositions 32 and 39. We choose such a pair of subsets as follows:

- We first choose $1 \leqslant i<j \leqslant n$ and will have $i=\max (I \backslash J)$ and $j=\min (J \backslash I)$ (to fulfill Proposition 39 (i)).

- For all $i<k<j$, we must have $k \in I \cap J$ if $k \in \delta^{-}$and $k \in[n] \backslash(I \cup J)$ if $k \in \delta^{+}$(to fulfill Proposition 39 (iii)). This is impossible if $\delta_{k}=\boldsymbol{\otimes}$ (explaining the condition over the sum), and leaves two choices if $\delta_{k}=$ (1) (explaining the power of 2 ).

- For all $k<i$, we must have $k \in I$ if $\delta_{i} \in \delta^{+}$, and $k \notin J$ if $\delta_{i} \in \delta^{-}$(to fulfill Proposition 32). Moreover, $k \in I \backslash J$ implies $\delta_{i} \neq$ (1) (to fulfill Proposition 39 (ii)). Thus, $k$ must lie in

$$
\begin{aligned}
& -I \backslash J \text { if } \delta_{i}=\boldsymbol{\otimes}, \\
& -I \cap J \text { or } I \backslash J \text { if } \delta_{i}=\boldsymbol{\bigotimes}, \\
& -[n] \backslash(I \cup J) \text { or } I \backslash J \text { if } \delta_{i}=\boldsymbol{\bigotimes}, \\
& -I \cap J \text { or }[n] \backslash(I \cap J) \text { if } \delta_{i}=\text { (1). }
\end{aligned}
$$

Moreover, the choices in the last three cases are handled by the function $\Omega$.

- For $j<k$, the argument is symmetric. 


\subsection{Facets of types cones of permutree fans}

In view of the unique exchange property of the $\delta$-permutree fan $\mathcal{F}_{\delta}$, each pair of exchangeable rays of $\mathcal{F}_{\delta}$ yields a wall-crossing inequality for the type cone $\overline{\mathbb{T} C}\left(\mathcal{F}_{\delta}\right)$. However, not all pairs of exchangeable rays yield facet-defining inequalities of $\overline{\mathbb{T}} \mathbb{C}\left(\mathcal{F}_{\delta}\right)$. The characterization of the facets of $\overline{\mathbb{T}}\left(\mathcal{F}_{\delta}\right)$ is very similar to that of the exchangeable rays, only point (ii) slightly differs.

Proposition 45. The rays $\boldsymbol{r}(I)$ and $\boldsymbol{r}(J)$ define a facet of the type cone $\overline{\mathbb{T} C}\left(\mathcal{F}_{\delta}\right)$ if and only if, up to swapping the roles of $I$ and $J$,

(i) $i:=\max (I \backslash J)<\min (J \backslash I)=: j$,

(ii) $I \backslash J=\{i\}$ or $\delta_{i}=\bigotimes$ and $J \backslash I=\{j\}$ or $\delta_{j}=\bigotimes$,

(iii) $] i, j\left[\cap \delta^{-} \subseteq I \cap J \quad\right.$ and $\left.\quad\right] i, j\left[\cap \delta^{+} \cap I \cap J=\varnothing\right.$.

Example 46. For the decorations of Figures 7, 9 and 10 , the facets of $\overline{\mathbb{T}}\left(\mathcal{F}_{\Phi \oplus \otimes \Phi)}\right.$ correspond to the pairs of subsets $\{1,2\},\{1,3\},\{12,13\},\{12,23\},\{123,134\},\{123,234\}$, $\{13,23\},\{13,34\},\{134,234\},\{2,3\},\{23,34\},\{3,4\}$, while the facets of $\overline{\mathbb{T} C}\left(\mathcal{F}_{\mathbb{Q} \otimes \bowtie}\right)$ correspond to the pairs of subsets $\{1,234\},\{12,4\},\{123,124\},\{124,34\}$.

Example 47. Specializing Proposition 45, we recover that all pairs of exchangeable rays of $\mathcal{F}_{\delta}$ described in Example 41 define facets of the type cone $\overline{\mathbb{T}}\left(\mathcal{F}_{\delta}\right)$ when $\delta=\mathbb{D}^{n}$ or $\delta=\bigotimes^{n}$. In contrast, when $\delta=\bigotimes^{n}$, only the pairs of intervals $\{[i, j[] i, j]$,$\} for some$ $1 \leqslant i<j \leqslant n$ correspond to facets of the type cone $\overline{\mathbb{T} C}\left(\mathcal{F}_{\mathbb{Q}^{n}}\right)$ (equivalently, the internal diagonals $(i-1, j)$ and $(i, j+1)$ of the $(n+2)$-gon that just differ by a shift).

Remark 48. Observe that combining the characterization of Proposition 32 with the condition of Proposition 45 (ii) implies that

- $\left[1, i\left[\right.\right.$ is included in $I \backslash J$ if $\delta_{i}=\boldsymbol{\otimes}$, in $[n] \backslash(I \cup J)$ if $\delta_{i}=\boldsymbol{\bigotimes}$, and in $I \cap J$ if $\delta_{i}=\boldsymbol{\bigotimes}$,

- $] j, n]$ is included in $J \backslash I$ if $\delta_{j}=\boldsymbol{\otimes}$, in $[n] \backslash(I \cup J)$ if $\delta_{j}=\boldsymbol{\bigotimes}$, and in $I \cap J$ if $\delta_{j}=\bigotimes$.

In particular, $I \backslash J$ is either $\{i\}$ or $[1, i]$ (and not both except if $i=1$ ), and $J \backslash I$ is either $\{j\}$ or $[j, n]$ (and not both except if $j=n$ ). The latter is however not equivalent to Proposition 45 (ii), since for instance the subsets $I=[1,2]$ and $J=[3]$ do not define a facet $\overline{\mathbb{T} C}\left(\mathcal{F}_{\otimes^{3}}\right)$.

Proof of Proposition 45. We consider two exchangeable rays $\boldsymbol{r}(I)$ and $\boldsymbol{r}(J)$, so that $I$ and $J$ satisfy the conditions of Propositions 32 and 39. We will show that they satisfy the additional condition of Proposition 45 (ii) if and only if the wall-crossing inequality corresponding to the exchange of $\boldsymbol{r}(I)$ and $\boldsymbol{r}(J)$ defines a facet of the type cone $\overline{\mathbb{T}} \mathbb{C}\left(\mathcal{F}_{\delta}\right)$.

Assume first that $I$ and $J$ do not satisfy Proposition 45 (ii). Since they satisfy Proposition 39 (ii), we have $|I \backslash J|>1$ and $\delta_{i} \in\{\bigotimes, \bigotimes\}$, or $|J \backslash I|>1$ and $\delta_{j} \in\{\bigotimes, \bigotimes\}$. 
Let us detail the case $|I \backslash J|>1$ and $\delta_{i}=\mathbb{Q}$, the other situations being symmetric. As usual, we define

$$
\begin{gathered}
i:=\max (I \backslash J), \quad j:=\min (J \backslash I), \\
D:=I \cap J, \quad U:=[n] \backslash(I \cup J), \quad L:=I \backslash J \backslash\{i\}, \quad R:=J \backslash I \backslash\{j\} .
\end{gathered}
$$

For each of the subsets $D, U, L$ and $R$, we choose an arbitrary permutree, and we construct $\delta$-permutrees $T$ and $S$ as in the proof of Proposition 39 and as illustrated in Figure 12, so that rotation from $T$ to $S$ exchanges $\boldsymbol{r}(I)$ to $\boldsymbol{r}(J)$. Note that we voluntarily placed $R$ at the same level as node $j$, as it can be the right ancestor or descendant subtree of $j$, depending on $\delta_{j}$. We know that the wall-crossing inequality corresponding to this rotation is

$$
h(I)+h(J)>h(I \cap J)+h(I \cup J) .
$$

Consider now the $\delta$-permutrees $V$ and $W$ of Figure 12. In the tree $V$ (resp. $W$ ), the leftmost lower blossom of $L$ is connected to $j$ (resp. $i$ ), and the rightmost upper blossom of $L$ is connected to the blossom of $U$ to which $j$ was connected in $T$. Note that these are indeed $\delta$-permutrees since $\delta_{i}=\bigotimes$. The rotation between $V$ and $W$ yields the wall-crossing inequality

$$
h\left(I^{\prime}\right)+h(J)>h(I \cap J)+h\left(I^{\prime} \cup J\right),
$$

where $I^{\prime}:=\{i\} \cup D=\{i\} \cup(I \cap J)$. Note that we could also have checked that $I^{\prime}$ and $J$ satisfy the conditions of Propositions 32 and 39.

Consider now the $\delta$-permutrees $X$ and $Y$ of Figure 12. We have already seen that $Y$ is indeed a $\delta$-permutree because it is just equal to $V$. The same arguments show that $X$ is also a $\delta$-permutree. Consider now the rotation of the edge joining $L$ to $j$. If $i$ and $j$ are connected to the same node in $L$, then this rotation gives the $\delta$-permutree $Y$. Otherwise, this rotation moves a part of $L$ in between $j$ and $U$ and leaves the remaining part of $L$ in between $i$ and $j$. Rotating again and again the edge between this remaining part of $L$ and $j$, we finally obtain the $\delta$-permutree $Y$. More formally, we can consider the sequence of rotations between the $\delta$-permutrees $X_{k}$ and $Y_{k}$ illustrated in Figure 12, where at each step we use $X_{k+1}=Y_{k}$. In these $\delta$-permutrees, we have $\underline{L}_{k} \sqcup \bar{L}_{k}=L$. Moreover, $\underline{L}_{k+1}$ is obtained from $\underline{L}_{k}$ by deleting the node connected to $j$ in $X_{k}$ and all its left ancestors and descendants. Hence, starting with $X_{0}=X$, we will end with $Y_{p}=Y$. Summing the wall-crossing inequalities corresponding to the rotations between $X_{k}$ and $Y_{k}$, we thus obtain the inequality

$$
h(I)+h\left(J^{\prime}\right)>h\left(I \cap J^{\prime}\right)+h(I \cup J),
$$

where $J^{\prime}:=\{i\} \cup J$.

Finally, observe that $I^{\prime}=I \cap J^{\prime}$ and $J^{\prime}=I^{\prime} \cup J$. We therefore obtain that the wallcrossing inequality (1) can be expressed as the sum of inequalities (2) and (3), showing that $I$ and $J$ do not define a facet of the type cone $\overline{\mathbb{T} C}\left(\mathcal{F}_{\delta}\right)$.

Conversely, assume that $I$ and $J$ satisfy the conditions of Proposition 45. To prove that the wall-crossing inequality associated with $\{I, J\}$ indeed defines a facet of the type cone $\overline{\mathbb{T C}}\left(\mathcal{F}_{\delta}\right)$, we exhibit a point $\boldsymbol{p}$ that satisfies the wall-crossing inequality associated 


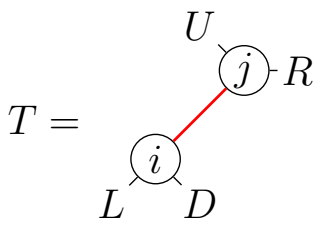<smiles>[R]C(=O)C=C</smiles><smiles>[CH]1C=C1</smiles><smiles></smiles><smiles></smiles><smiles>[X]C=CC(C)(Br)Br</smiles><smiles>[C]1C=CC=C1</smiles><smiles></smiles><smiles>[R]C(C)C1=CC=C1</smiles>

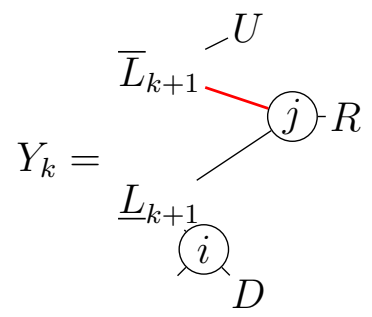

Figure 12: Some rotations in $\delta$-permutrees. The third column is a combination of rotations of the form given in the fourth column.

with any pair $\{K, L\}$ fulfilling the conditions of Proposition 45, except for the one associated with the pair $\{I, J\}$.

For this, we need some notations and definitions. We denote by $\wp(A):=\{X \subseteq A\}$ the set of subsets of a set $A$, and define $\nabla(A, B):=\wp(A \cup B) \backslash(\wp(A) \cup \wp(B))$ for two sets $A, B$. For a pair $\{A, B\}$ with $A \backslash B \neq \varnothing \neq B \backslash A$, observe that

- $A \cup B$ is the inclusion-maximal element of $\nabla(A, B)$,

- $A$ and $B$ are the inclusion-maximal subset of $A \cup B$ not in $\nabla(A, B)$,

- the pairs in $\nabla(A, B)$ are precisely the pairs $\{a, b\}$ for $a \in A \backslash B$ and $b \in B \backslash A$.

This implies that for two pairs $\{A, B\}$ and $\{C, D\}$ such that $A \backslash B \neq \varnothing \neq B \backslash A$ and $C \backslash D \neq \varnothing \neq D \backslash C$,

- if $\nabla(A, B)=\nabla(C, D)$, then $\{A, B\}=\{C, D\}$,

- if $\nabla(A, B) \subseteq \nabla(C, D)$, then $A \cup B \subseteq C \cup D$, and up to reversing the roles of $C$ and $D, A \backslash B \subseteq C \backslash D$ and $A \backslash B \subseteq C \backslash D$.

We denote by $\left(\boldsymbol{g}_{M}\right)_{M \in \mathcal{I}}$ the standard basis of the space $\mathbb{R}^{\mathcal{I}}$ indexed by the rays $\mathcal{I}$ of $\mathcal{F}_{\delta}$. Let $\boldsymbol{n}(I, J):=\boldsymbol{g}_{I}+\boldsymbol{g}_{J}-\boldsymbol{g}_{I \cap J}-\boldsymbol{g}_{I \cup J}$ denote the normal vector of the wall-crossing inequality associated with our pair $\{I, J\}$ of subsets. We are thus looking for a point $\boldsymbol{p} \in \mathbb{R}^{\mathcal{I}}$ such that $\langle\boldsymbol{p} \mid \boldsymbol{n}(I, J)\rangle<0$ while $\langle\boldsymbol{p} \mid \boldsymbol{n}(K, L)\rangle \geqslant 0$ for all pairs $\{K, L\}$ that fulfill the conditions of Proposition 45 and are distinct from $\{I, J\}$. To find such a point, we define 
three vectors $\boldsymbol{x}, \boldsymbol{y}, \boldsymbol{z} \in \mathbb{R}^{\mathcal{I}}$ given by

$\boldsymbol{x}:=-\sum_{M \in \mathcal{I}}|\wp(M) \backslash \nabla(I, J)| \boldsymbol{g}_{M}, \quad \boldsymbol{y}:=-\sum_{M \in \mathcal{I}}|\wp(M) \cap \nabla(I, J)| \boldsymbol{g}_{M}, \quad$ and $\quad \boldsymbol{z}:=-\boldsymbol{n}(I, J)$, and we consider the point $\boldsymbol{p} \in \mathbb{R}^{\mathcal{I}}$ defined by

$$
\boldsymbol{p}:=\lambda \boldsymbol{x}+\mu \boldsymbol{y}+\boldsymbol{z},
$$

where $\lambda$ and $\mu$ are arbitrary scalars such that $\lambda>|\langle\boldsymbol{z} \mid \boldsymbol{n}(K, L)\rangle|$ for any pair $\{K, L\}$ of subsets and $0<\mu|\nabla(I, J)|<\langle\boldsymbol{z} \mid \boldsymbol{z}\rangle$. We will prove that the point $\boldsymbol{p}$ satisfies the desired inequalities.

For this, consider a pair $\{K, L\}$ that fulfills the conditions of Proposition 45. Observe that

$$
\begin{aligned}
\langle\boldsymbol{x} \mid \boldsymbol{n}(K, L)\rangle= & \boldsymbol{x}_{K}+\boldsymbol{x}_{L}-\boldsymbol{x}_{K \cap L}-\boldsymbol{x}_{K \cup L} \\
= & -|\wp(K) \backslash \nabla(I, J)|-|\wp(L) \backslash \nabla(I, J)| \\
& +|\wp(K \cup L) \backslash \nabla(I, J)|+|\wp(K \cap L) \backslash \nabla(I, J)| \\
= & |\nabla(K, L) \backslash \nabla(I, J)|,
\end{aligned}
$$

by inclusion-exclusion principle. Similarly

$$
\langle\boldsymbol{y} \mid \boldsymbol{n}(K, L)\rangle=|\nabla(K, L) \cap \nabla(I, J)| .
$$

Finally,

$$
\begin{aligned}
\langle\boldsymbol{z} \mid \boldsymbol{n}(K, L)\rangle & =-\langle\boldsymbol{n}(I, J) \mid \boldsymbol{n}(K, L)\rangle \\
& =-\left\langle\boldsymbol{g}_{I}+\boldsymbol{g}_{J}-\boldsymbol{g}_{I \cup J}-\boldsymbol{g}_{I \cap J} \mid \boldsymbol{g}_{K}+\boldsymbol{g}_{L}-\boldsymbol{g}_{K \cup L}-\boldsymbol{g}_{K \cap L}\right\rangle
\end{aligned}
$$

is a signed sum of Kronecker deltas $\delta_{X, Y}$ where $X$ ranges over $\{I, J, I \cup J, I \cap J\}$ and $Y$ ranges over $\{K, L, K \cup L, K \cap L\}$. We leave to the end of the proof the claim that these two sets are disjoint when $\nabla(K, L) \subsetneq \nabla(I, J)$, so that $\langle\boldsymbol{z} \mid \boldsymbol{n}(K, L)\rangle=0$.

We therefore obtain that

$$
\langle\boldsymbol{p} \mid \boldsymbol{n}(I, J)\rangle=\mu|\nabla(I, J)|-\langle\boldsymbol{z} \mid \boldsymbol{z}\rangle<0
$$

since $\mu|\nabla(I, J)|<\langle\boldsymbol{z} \mid \boldsymbol{z}\rangle$, while

$$
\langle\boldsymbol{p} \mid \boldsymbol{n}(K, L)\rangle=\lambda|\nabla(K, L) \backslash \nabla(I, J)|+\mu|\nabla(K, L) \cap \nabla(I, J)|-\langle\boldsymbol{z} \mid \boldsymbol{n}(K, L)\rangle \geqslant 0
$$

for any other pair $\{K, L\}$ that fulfills the conditions of Proposition 45. Indeed,

- if $\nabla(K, L) \nsubseteq \nabla(I, J)$, then $\lambda|\nabla(K, L) \backslash \nabla(I, J)| \geqslant \lambda$ so that $\lambda|\nabla(K, L) \backslash \nabla(I, J)|-$ $\langle\boldsymbol{z} \mid \boldsymbol{n}(K, L)\rangle>0$ and $\mu|\nabla(K, L) \cap \nabla(I, J)| \geqslant 0$,

- if $\nabla(K, L) \subsetneq \nabla(I, J)$, then $\lambda|\nabla(K, L) \backslash \nabla(I, J)|=0$ while $\mu|\nabla(K, L) \cap \nabla(I, J)|>\mu$ and we claimed that $\langle\boldsymbol{z} \mid \boldsymbol{n}(K, L)\rangle=0$. 
Finally, we conclude by proving our claim that if $\nabla(K, L) \subsetneq \nabla(I, J)$, then the sets $\{I, J, I \cup J, I \cap J\}$ and $\{K, L, K \cup L, K \cap L\}$ are disjoint. Up to reversing the roles of $I$ and $J$ (resp. $K$ and $L$ ), we assume that $i:=\max (I \backslash J)<\min (J \backslash I)=: j$ (resp. $k:=\max (K \backslash L)<\min (L \backslash K)=: \ell$ ). As already observed earlier, the inclusion $\nabla(K, L) \subsetneq \nabla(I, J)$ implies the inclusions $K \backslash L \subseteq I \backslash J$ and $L \backslash K \subseteq J \backslash I$, so that $k \leqslant i<j \leqslant \ell$. It turns out that the conditions of Proposition 45 actually imply that $K \backslash L=I \backslash J$ and $L \backslash K=J \backslash I$, so that $k=i$ and $j=\ell$. Indeed,

- if $I \backslash J=\{i\}$, then $K \backslash L=\{i\}$ (since $\varnothing \neq K \backslash L \subseteq I \backslash J)$,

- if $\delta_{i}=\bigotimes$, then $K \backslash L=I \backslash J=[1, i]$ (as otherwise $\left.i \in\right] k, \ell\left[\cap \delta^{-} \cap \delta^{+}\right.$).

(The argument is symmetric for the equality $L \backslash K=J \backslash I$.) Observe now that

- if $I \cap J=K \cap L$, then $I=(I \cap J) \cup(I \backslash J)=(K \cap L) \cup(K \backslash L)=K$ and similarly $J=L$,

- if $I \cup J=K \cup L$, then $I=(I \cup J) \backslash(J \backslash I)=(K \cup L) \backslash(L \backslash K)=K$ and similarly $J=L$,

- if $I=K$, then $J=(J \backslash I) \cup(I \backslash(I \backslash J))=(L \backslash K) \cup(K \backslash(K \backslash L))=L$,

- similarly, if $J=L$, then $I=K$.

In these four cases, we obtained that $I=K$ and $J=L$, which contradicts our assumption that $\nabla(K, L) \neq \nabla(I, J)$. Finally, observe that

- $I \notin\{L, K \cup L, K \cap L\}$ since $i \in I \backslash L$ and $j \in L \backslash I$. Similarly, $J \notin\{K, K \cup L, K \cap L\}$, $K \notin\{J, I \cup J, I \cap J\}$ and $L \notin\{J, I \cup J, I \cap J\}$.

- $I \cup J \neq K \cap L$ since $i \in I \backslash L$. Similarly, $I \cap J \neq K \cup L$.

We have thus checked that the sets $\{I, J, I \cup J, I \cap J\}$ and $\{K, L, K \cup L, K \cap L\}$ are disjoint, which ends the proof.

The proof of the following consequence of Proposition 45 is almost identical to that of Corollary 42.

Corollary 49. The number $\phi(\delta)$ of facets of the type cone $\overline{\mathbb{T C}}\left(\mathcal{F}_{\delta}\right)$ of the $\delta$-permutree fan $\mathcal{F}_{\delta}$ is

$$
\phi(\delta)=\sum_{\substack{1 \leqslant i<j \leqslant n \\ \forall i<k<j, \delta_{k} \neq \otimes}} \Omega\left(\delta_{1} \ldots \delta_{i-1}\right)^{\delta_{i}=\Phi} \cdot 2^{\left|\left\{i<k<j \mid \delta_{k}=\mathbb{\Phi}\right\}\right|} \cdot \Omega\left(\delta_{n} \ldots \delta_{j+1}\right)^{\delta_{j}=\mathbb{\Phi},}
$$

where $\Omega\left(\delta_{1} \ldots \delta_{k}\right)$ is defined inductively from $\Omega(\varepsilon)=1$ by

$$
\Omega\left(\delta_{1} \ldots \delta_{k}\right)= \begin{cases}2 \cdot \Omega\left(\delta_{1} \ldots \delta_{k-1}\right) & \text { if } \delta_{k}=\mathbb{D} \\ 1+\Omega\left(\delta_{1} \ldots \delta_{k-1}\right) & \text { if } \delta_{k} \in\{\boldsymbol{Q}, \boldsymbol{\bigotimes}\} \\ 2 & \text { if } \delta_{k}=\boldsymbol{\otimes}\end{cases}
$$


Example 50. For the decorations of Figures 7, 9 and 10, we obtain $\phi(D \emptyset \otimes()=12$ and $\phi(1 \otimes \otimes(1)=4$.

Example 51. Specializing the formula of Corollary 49, we recover the following classical numbers:

- when $\delta=\mathbb{D}^{n}$, the type cone $\overline{\mathbb{T} C}\left(\mathcal{F}_{\mathbb{D}^{n}}\right)$ has $2^{n-2}\left(\begin{array}{l}n \\ 2\end{array}\right)$ facets,

- when $\delta=\mathbb{Q}^{n}$, the type cone $\overline{\mathbb{T} C}\left(\mathcal{F}_{\bigotimes^{n}}\right)$ has $\left(\begin{array}{l}n \\ 2\end{array}\right)$ facets (equalling the number of squares of the form $(i-1, i, j, j+1)$ in the $(n+2)$-gon),

- when $\delta=\bigotimes^{n}$, the type cone $\overline{\mathbb{T}}\left(\mathcal{F}_{\bigotimes^{n}}\right)$ has $n-1$ facets.

Corollary 52. If $\delta \in\{\otimes, \bigotimes, \otimes\}^{n}$, we have

$$
\phi(\delta)=\left|\left\{1 \leqslant i<j \leqslant n \mid \forall i<k<j, \delta_{k} \neq \otimes\right\}\right| .
$$

Corollary 53. The type cone $\overline{\mathbb{T}}\left(\mathcal{F}_{\delta}\right)$ is simplicial if and only if $\delta_{k} \neq \mathbb{D}$ for any $\left.k \in\right] 1, n[$.

Proof. The type cone $\overline{\mathbb{T C}}\left(\mathcal{F}_{\delta}\right)$ is simplicial if and only if the number $\rho(\delta)$ of rays of $\mathcal{F}_{\delta}$ and the number $\phi(\delta)$ of facets of $\overline{\mathbb{T} C}\left(\mathcal{F}_{\delta}\right)$ satisfy the equality $\rho(\delta)=\phi(\delta)+n-1$. Considering the formulas of Corollary 35 for $\rho(\delta)$ and of Corollary 49 for $\phi(\delta)$, we observe that $\rho(\delta)=\phi(\delta)+n-1$ if and only if $\psi(i, j):=\Omega\left(\delta_{1} \ldots \delta_{i-1}\right)^{\delta_{i}=\mathbb{D}} \cdot \Omega\left(\delta_{n} \ldots \delta_{j+1}\right)^{\delta_{j}=\mathbb{C}}$ is always equal to 1 in the formula for $\phi(\delta)$. It is clearly the case if $\delta_{k} \neq$ (1) for any $k \in] 1, n\left[\right.$. Conversely, if $\delta_{k}=\mathbb{( 1 )}$ for some $\left.k \in\right] 1, n[$, then we have $\psi(k-1, k) \geqslant 2$ (and also $\psi(k, k+1) \geqslant 2$ ), so that the equality does not hold.

\subsection{Kinematic permutreehedra}

Applying Proposition 3, we obtain the following realizations of the $\delta$-permutree fans in the kinematic space [AHBHY18] when $\delta \in\{\boldsymbol{Q}, \boldsymbol{\bigotimes}, \boldsymbol{\otimes}\}^{n}$. To simplify our statement, we assume that $\delta_{1}=\delta_{n}=\bigotimes\left(\right.$ this assumption does not lose generality as the decorations $\delta_{1}$ and $\delta_{n}$ are irrelevant in all constructions). Consider the sets

$$
\mathfrak{F}:=\left\{1 \leqslant i<j \leqslant n \mid \forall i<k<j, \delta_{k} \neq \boldsymbol{\bigotimes}\right\} \quad \text { and } \quad \mathfrak{R}:=\{0,1\} \times[n]^{2} \times\{0,1\}
$$

and define $p_{i, j}^{\varepsilon}$ and $q_{i, j}^{\varepsilon}$ for $(i, j) \in \mathfrak{F}$ and $\varepsilon \in\{+,-\}$ by

$$
\begin{aligned}
& p_{i, j}^{\varepsilon}:= \begin{cases}\min \left(\{j\} \cup(] i, j\left[\cap \delta^{\varepsilon}\right)\right)-1 & \text { if } i \in \delta^{\varepsilon}, \\
i-1 & \text { if } i \notin \delta^{\varepsilon},\end{cases} \\
& q_{i, j}^{\varepsilon}:= \begin{cases}\max \left(\{i\} \cup(] i, j\left[\cap \delta^{\varepsilon}\right)\right)+1 & \text { if } j \in \delta^{\varepsilon}, \\
j+1 & \text { if } j \notin \delta^{\varepsilon} .\end{cases}
\end{aligned}
$$

Using these notations, we obtain the following realizations of the $\delta$-permutree fan. 
Corollary 54. Let $\delta \in\{\otimes, \bigotimes, \bigotimes\}^{n}$ with $\delta_{1}=\delta_{n}=\bigotimes$, and the notations introduced above. Then, for any $\boldsymbol{u} \in \mathbb{R}_{>0}^{\mathfrak{F}}$, the polytope $Q_{\delta}(\boldsymbol{u})$ defined as the set $\boldsymbol{z} \in \mathbb{R}_{\geqslant 0}^{\Re}$ such that

- $\boldsymbol{z}_{(\ell, p, q, r)}=0$ if $(p, q) \notin \mathfrak{F}$,

- $\boldsymbol{z}_{(\ell, p, q, r)}=\boldsymbol{z}_{\left(\ell^{\prime}, p, q, r^{\prime}\right)}$ if $p+1 \neq q$,

- and for all $(i, j) \in \mathfrak{F}$,

$$
\boldsymbol{z}_{\left(1, p_{i, j}^{+}, q_{i, j}^{-}, 0\right)}+\boldsymbol{z}_{\left(0, p_{i, j}^{-}, q_{i, j}^{+}, 1\right)}-\boldsymbol{z}_{\left(i \notin \delta^{-}, p_{i, j+1}^{-}, q_{i-1, j}^{-}, j \notin \delta^{-}\right)}-\boldsymbol{z}_{\left(i \in \delta^{+}, p_{i, j+1}^{+}, q_{i-1, j}^{+}, j \in \delta^{+}\right)}=\boldsymbol{u}_{(i, j)}
$$

is a $\delta$-permutreehedron, whose normal fan is the $\delta$-permutree fan $\mathcal{F}_{\delta}$. Moreover, the polytopes $Q_{\delta}(\boldsymbol{u})$ for $\boldsymbol{u} \in \mathbb{R}_{>0}^{\mathfrak{F}}$ describe all polytopal realizations of the $\delta$-permutree fan $\mathcal{F}_{\delta}$.

Example 55. Specializing the construction of Corollary 54, we obtain:

- when $\delta=\otimes \bigotimes^{n-2} \otimes$, we have

$$
p_{i, j}^{-}=\left\{\begin{array}{ll}
j-1 & \text { if } i=1, \\
i-1 & \text { if } i \neq 1,
\end{array} \quad p_{i, j}^{+}=i, \quad q_{i, j}^{-}=\left\{\begin{array}{ll}
i+1 & \text { if } j=n, \\
j+1 & \text { if } j \neq n,
\end{array} \quad \text { and } \quad q_{i, j}^{+}=j,\right.\right.
$$

so that the polytope $Q_{\delta}(\boldsymbol{u})$ is equivalent to the kinematic associahedron defined in [AHBHY18]:

$$
\left\{\begin{array}{l|l}
\boldsymbol{y} \in \mathbb{R}^{\left(\begin{array}{c}
{[0, n+1]} \\
2
\end{array}\right)} \mid \begin{array}{l}
\boldsymbol{y} \geqslant 0, \quad \boldsymbol{y}_{(i, j)}=0 \text { if } i+1=j, \quad \boldsymbol{y}_{(0, n+1)}=0, \quad \text { and } \\
\boldsymbol{y}_{(i, j+1)}+\boldsymbol{y}_{(i-1, j)}-\boldsymbol{y}_{(i-1, j+1)}-\boldsymbol{y}_{(i, j)}=\boldsymbol{u}_{(i, j)} \text { for all }(i, j) \in\left(\begin{array}{c}
{[n]} \\
2
\end{array}\right)
\end{array}
\end{array}\right\} .
$$

(The map is given by $\boldsymbol{y}_{(0, j)}=\boldsymbol{z}_{(1, j-1, j, 0)}, \boldsymbol{y}_{(i, n+1)}=\boldsymbol{z}_{(0, i, i+1,1)}$ and $\boldsymbol{y}_{(i, j)}=\boldsymbol{z}_{(\ell, i, j, r)}$ for any $\ell, r \in\{0,1\}$.)

- when $\delta=\bigotimes^{n}$, we have

$$
p_{i, j}^{-}=p_{i, j}^{+}=i \quad \text { and } \quad q_{i, j}^{-}=q_{i, j}^{+}=j,
$$

so that the polytope $Q_{\delta}(\boldsymbol{u})$ is equivalent to the following kinematic cube:

$$
\left\{\boldsymbol{y} \in \mathbb{R}^{\{0,1\} \times[n-1]} \mid \boldsymbol{y} \geqslant 0 \quad \text { and } \quad \boldsymbol{y}_{(0, i)}+\boldsymbol{y}_{(1, i)}=\boldsymbol{u}_{(i, i+1)} \text { for all } i \in[n-1]\right\}
$$

(The map is given by $\boldsymbol{y}_{(0, i)}=\boldsymbol{z}_{(0, i, i+1,1)}$ and $\boldsymbol{y}_{(1, i)}=\boldsymbol{z}_{(1, i, i+1,0)}$.)

Proof of Corollary 54. According to Corollaries 38 and 52, we can parametrize

1. the rays of the fan $\mathcal{F}_{\delta}$ by $\mathfrak{R}$ where we identify $(0, p, q, 0),(1, p, q, 0),(0, p, q, 1)$ and $(1, p, q, 1)$ when $p+1 \neq q$, and ignore $(0, p, q, 0),(1, p, q, 0),(0, p, q, 1)$ and $(1, p, q, 1)$ for $(p, q) \notin \mathfrak{F}$.

2. the facets of its type cone $\overline{\mathbb{T} C}\left(\mathcal{F}_{\delta}\right)$ by $F$. 
To be more precise, with any $(\ell, p, q, r) \in \mathfrak{R}$, we associate the subset $R_{(\ell, p, q, x)}$ of $[n]$ defined as follows:

- if $(p, q) \notin \mathfrak{F}$ then $R_{(0, p, q, 0)}:=R_{(1, p, q, 0)}:=R_{(0, p, q, 1)}:=\varnothing$ while $R_{(1, p, q, 1)}:=[n]$,

- if $p+1=q$, then $R_{(0, p, q, 0)}:=\varnothing, R_{(1, p, q, 0)}:=[1, p], R_{(0, p, q, 1)}:=[q, n]$ and $R_{(1, p, q, 1)}:=[n]$,

- if $(p, q) \in \mathfrak{F}$ and $p+1 \neq q$, then independently of the values of $\ell$ and $r$, the set $R_{(\ell, p, q, r)}$ is the unique proper subset $\varnothing \neq R \subsetneq[n]$ which fulfills the conditions of Proposition 32 and with the property that $i$ is the last position (resp. $j$ is the first position) such that $1, \ldots, i$ (resp. $j, \ldots, n$ ) all belong to $R$ or all belong to $[n] \backslash R$, (see the proof of Corollary 35 for the uniqueness of $R$ ).

Now with any $(i, j) \in \mathfrak{F}$, we associate the unique pair $\left(I_{i, j}, J_{i, j}\right)$ of proper subsets satisfying the conditions of Propositions 32 and 45 and such that $\max \left(I_{i, j} \backslash J_{i, j}\right)=i$ and $\min \left(J_{i, j} \backslash I_{i, j}\right)=j$ (see Remark 48 to argue that such a pair is unique). We claim that these sets are given by

$$
I_{i, j}=R_{\left(1, p_{i, j}^{+}, q_{i, j}^{-}, 0\right)} \quad \text { and } \quad J_{i, j}=R_{\left(0, p_{i, j}^{-}, q_{i, j}^{+}, 1\right)},
$$

and their intersection and union are given by

$$
I_{i, j} \cap J_{i, j}=R_{\left(i \notin \delta^{-}, p_{i, j+1}^{-}, q_{i-1, j}^{-}, j \notin \delta^{-}\right)} \quad \text { and } \quad I_{i, j} \cup J_{i, j}=R_{\left(i \in \delta^{+}, p_{i, j+1}^{+}, q_{i-1, j}^{+}, j \in \delta^{+}\right)},
$$

which implies the result by Propositions 3 and 30 .

To show this claim, let us first prove that $I_{i, j}=R_{\left(1, p_{i, j}^{+}, q_{i, j}^{-}, 0\right)}$. Recall from Proposition 45 (iii) that $] i, j\left[\cap \delta^{-} \subseteq I_{i, j}\right.$ and $] i, j\left[\cap \delta^{+} \subseteq[n] \backslash I_{i, j}\right.$. Moreover, we obtain by Remark 48 that

- if $i \in \delta^{+}$, then $[1, i] \subseteq I_{i, j}$ and the last position $p$ for which $1, \ldots, p$ all belong to $I_{i, j}$ is the position just before the first element in $] i, j\left[\cap \delta^{+}\right.$, or $j$ if there is no such element,

- otherwise, $\left[1, i\left[\subseteq[n] \backslash I_{i, j}\right.\right.$ while $i \in I_{i, j}$, so that the last position $p$ for which $1, \ldots, p$ all belong to $[n] \backslash I_{i, j}$ is $i-1$.

This shows that $p_{i, j}^{+}$indeed gives the last position $p$ for which $1, \ldots, p$ all belong to $I_{i, j}$ or all belong to $[n] \backslash I_{i, j}$. A symmetric argument shows that $q_{i, j}^{-}$gives the first position $q$ for which $q, \ldots, n$ all belong to $I_{i, j}$ or all belong to $[n] \backslash I_{i, j}$. This ensures that $1 \leqslant p_{i, j}^{+}<q_{i, j}^{-} \leqslant n$. Moreover, we have $\delta_{k} \neq \otimes$ for $p_{i, j}^{+}<k<q_{i, j}^{-}$, since $i-1 \leqslant p_{i, j}^{+}$ with equality only when $i \notin \delta^{+}$and $q_{i, j}^{-} \leqslant j+1$ with equality only when $j \notin \delta^{-}$. Thus, $\left(p_{i, j}^{+}, q_{i, j}^{-}\right) \in \mathfrak{F}$.

Observe now that if $i \notin \delta^{+}$, then $p_{i, j}^{+}=i-1$ while $q_{i, j}^{-} \geqslant i+1$ so that $p_{i, j}^{+}+1<q_{i, j}^{-}$. We therefore obtain that

- if $p_{i, j}^{+}+1=q_{i, j}^{-}$, then $i \in \delta^{+}$so that $[1, i] \subseteq I_{i, j}$ and thus $I_{i, j}=\left[1, p_{i, j}^{+}\right]=R_{\left(1, p_{i, j}^{+}, q_{i, j}^{-}, 0\right)}$, 
- if $p_{i, j}^{+}+1<q_{i, j}^{-}$, then $I_{i, j}=R_{\left(1, p_{i, j}^{+}, q_{i, j}^{-}, 0\right)}$ as they are both fully determined by $p_{i, j}^{+}$ and $q_{i, j}^{-}$.

This concludes our proof of $I_{i, j}=R_{\left(1, p_{i, j}^{+}, q_{i, j}^{-}, 0\right)}$. By symmetry $J_{i, j}=R_{\left(0, p_{i, j}^{-}, q_{i, j}^{+}, 1\right)}$.

For the intersection, note first that if $I_{i, j} \cap J_{i, j}=\varnothing$, then $i, j \in \delta^{-}$by Remark 48 while $] i, j\left[\subseteq \delta^{+}\right.$by Proposition 45 (iii). Therefore, $p_{i, j+1}^{-}=j-1$ and $q_{i-1, j}^{-}=i+1$, which implies that $\left(p_{i, j+1}^{-} \nless q_{i-1, j}^{-}\right) \notin \mathfrak{F}$ or $p_{i, j+1}^{-}+1=q_{i-1, j}^{-}$. Since $i, j \in \delta^{-}$, this yields in both situations that $R_{\left(i \notin \delta^{-}, p_{i, j+1}^{-}, q_{i-1, j}^{-}, j \notin \delta^{-}\right)}=\varnothing=I_{i, j} \cap J_{i, j}$.

Assume now that $I_{i, j} \cap J_{i, j} \neq \varnothing$. We then obtain by Remark 48 that

- if $i \in \delta^{+}$, then $[1, i] \subseteq[n] \backslash\left(I_{i, j} \cap J_{i, j}\right)$ and the last position $p$ for which $1, \ldots, p$ all belong to $[n] \backslash\left(I_{i, j} \cap J_{i, j}\right)$ is the position just before the first element in $] i, j\left[\cap \delta^{-}\right.$, or $j+1$ if there is no such element,

- otherwise, $\left[1, i\left[\subseteq I_{i, j} \cap J_{i, j}\right.\right.$ while $i \notin I_{i, j} \cap J_{i, j}$, so that the last position $p$ for which $1, \ldots, p$ all belong to $I_{i, j} \cap J_{i, j}$ is $i-1$.

This shows that $p_{i, j+1}^{-}$indeed gives the last position $p$ for which $1, \ldots, p$ belongs all to $I_{i, j} \cap J_{i, j}$ or all to $[n] \backslash\left(I_{i, j} \cap J_{i, j}\right)$. A symmetric argument shows that $q_{i-1, j}^{-}$gives the first position $q$ for which $q, \ldots, n$ belongs all to $I_{i, j} \cap J_{i, j}$ or all to $[n] \backslash\left(I_{i, j} \cap J_{i, j}\right)$. As above, this ensures that $\left(p_{i, j+1}^{-}, q_{i-1, j}^{-}\right) \in \mathfrak{F}$.

We now distinguish three cases:

- if $p_{i, j+1}^{-}+1=q_{i-1, j}^{-}$and $\left[1, i\left[\subseteq I_{i, j} \cap J_{i, j}\right.\right.$, then $i \in \delta^{+}$and $j \in \delta^{-}$by Remark 48, so that we get $I_{i, j} \cap J_{i, j}=\left[1, p_{i, j+1}^{-}\right]=R_{\left(0, p_{i, j+1}^{-}, q_{i-1, j}^{-}, 1\right)}=R_{\left(i \notin \delta^{-}, p_{i, j+1}^{-}, q_{i-1, j}^{-}, j \notin \delta^{-}\right)}$.

- if $p_{i, j+1}^{-}+1=q_{i-1, j}^{-}$and $[1, i] \subseteq[n] \backslash\left(I_{i, j} \cap J_{i, j}\right)$, then $i \in \delta^{-}$and $j \in \delta^{+}$by Remark 48, so that we get $I_{i, j} \cap J_{i, j}=\left[q_{i-1, j}^{-}, n\right]=R_{\left(1, p_{i, j+1}^{-}, q_{i-1, j}^{-}, 0\right)}=R_{\left(i \notin \delta^{-}, p_{i, j+1}^{-}, q_{i-1, j}^{-}, j \notin \delta^{-}\right)}$.

- if $p_{i, j+1}^{-}+1<q_{i-1, j}^{-}$, then $\left.I_{i, j} \cap J_{i, j}=R_{\left(i \notin \delta^{-}, p_{i, j+1}^{-}, q_{i-1, j}^{-}\right.}, j \notin \delta^{-}\right)$as they are both fully determined by $p_{i, j+1}^{-}$and $q_{i-1, j}^{-}$.

This concludes the proof for the intersection $I_{i, j} \cap J_{i, j}=R_{\left(i \notin \delta^{-}, p_{i, j+1}^{-}, q_{i-1, j}^{-}, j \notin \delta^{-}\right)}$. The proof for the union $I_{i, j} \cup J_{i, j}=R_{\left(i \in \delta^{+}, p_{i, j+1}^{+}, q_{i-1, j}^{+}, j \in \delta^{+}\right)}$is identical.

\section{Acknowledgements}

We are grateful to J.-C. Novelli for multiple discussions and suggestions, to N. Reading for bibliographic inputs, to A. Padrol for comments on a preliminary version, and to an anonymous referee for various suggestions on the presentation of this paper.

\section{References}

[ACEP20] Federico Ardila, Federico Castillo, Christopher Eur, and Alexander Postnikov. Coxeter submodular functions and deformations of Coxeter permutahedra. Adv. Math., 365:107039, 36, 2020. 
[AHBHY18] Nima Arkani-Hamed, Yuntao Bai, Song He, and Gongwang Yan. Scattering forms and the positive geometry of kinematics, color and the worldsheet. $J$. High Energy Phys., (5):096, front matter+75, 2018.

[CD06] Michael P. Carr and Satyan L. Devadoss. Coxeter complexes and graphassociahedra. Topology Appl., 153(12):2155-2168, 2006.

[CFZ02] Frédéric Chapoton, Sergey Fomin, and Andrei Zelevinsky. Polytopal realizations of generalized associahedra. Canad. Math. Bull., 45(4):537-566, 2002.

[CP17] Grégory Chatel and Vincent Pilaud. Cambrian Hopf Algebras. Adv. Math., 311:598-633, 2017.

[Dev09] Satyan L. Devadoss. A realization of graph associahedra. Discrete Math., 309(1):271-276, 2009.

[DRS10] Jesus A. De Loera, Jörg Rambau, and Francisco Santos. Triangulations: Structures for Algorithms and Applications, volume 25 of Algorithms and Computation in Mathematics. Springer Verlag, 2010.

[GKZ08] Israel Gelfand, Mikhail Kapranov, and Andrei Zelevinsky. Discriminants, resultants and multidimensional determinants. Modern Birkhäuser Classics. Birkhäuser Boston Inc., Boston, MA, 2008. Reprint of the 1994 edition.

[HL07] Christophe Hohlweg and Carsten Lange. Realizations of the associahedron and cyclohedron. Discrete Comput. Geom., 37(4):517-543, 2007.

[HNT05] Florent Hivert, Jean-Christophe Novelli, and Jean-Yves Thibon. The algebra of binary search trees. Theoret. Comput. Sci., 339(1):129-165, 2005.

[KT97] Daniel Krob and Jean-Yves Thibon. Noncommutative symmetric functions. IV. Quantum linear groups and Hecke algebras at $q=0$. J. Algebraic Combin., 6(4):339-376, 1997.

[Lod04] Jean-Louis Loday. Realization of the Stasheff polytope. Arch. Math. (Basel), 83(3):267-278, 2004.

[LP18] Carsten Lange and Vincent Pilaud. Associahedra via spines. Combinatorica, 38(2):443-486, 2018.

[LR98] Jean-Louis Loday and María O. Ronco. Hopf algebra of the planar binary trees. Adv. Math., 139(2):293-309, 1998.

[McM73] Peter McMullen. Representations of polytopes and polyhedral sets. Geometriae Dedicata, 2:83-99, 1973.

[Mey74] Walter Meyer. Indecomposable polytopes. Trans. Amer. Math. Soc., 190:7786, 1974.

[Nov00] Jean-Christophe Novelli. On the hypoplactic monoid. Discrete Math., 217(13):315-336, 2000. Formal power series and algebraic combinatorics (Vienna, 1997).

[Pil17] Vincent Pilaud. Which nestohedra are removahedra? Rev. Colombiana Mat., 51(1):21-42, 2017.

[Pil19] Vincent Pilaud. Hopf algebras on decorated noncrossing arc diagrams. J. Combin. Theory Ser. A, 161:486-507, 2019. 
[Pos09] Alexander Postnikov. Permutohedra, associahedra, and beyond. Int. Math. Res. Not. IMRN, (6):1026-1106, 2009.

[PP18] Vincent Pilaud and Viviane Pons. Permutrees. Algebraic Combinatorics, 1(2):173-224, 2018.

[PPPP19] Arnau Padrol, Yann Palu, Vincent Pilaud, and Pierre-Guy Plamondon. Associahedra for finite type cluster algebras and minimal relations between $\boldsymbol{g}$-vectors. Preprint, arXiv:1906.06861, 2019.

[PPR20] Arnau Padrol, Vincent Pilaud, and Julian Ritter. Quotientopes via minkowski sums of shard polytopes. In preparation, 2020.

[PRW08] Alexander Postnikov, Victor Reiner, and Lauren K. Williams. Faces of generalized permutohedra. Doc. Math., 13:207-273, 2008.

[PS19] Vincent Pilaud and Francisco Santos. Quotientopes. Bull. Lond. Math. Soc., 51(3):406-420, 2019.

[Rea03] Nathan Reading. Lattice and order properties of the poset of regions in a hyperplane arrangement. Algebra Universalis, 50(2):179-205, 2003.

[Rea04] Nathan Reading. Lattice congruences of the weak order. Order, 21(4):315344, 2004.

[Rea05] Nathan Reading. Lattice congruences, fans and Hopf algebras. J. Combin. Theory Ser. A, 110(2):237-273, 2005.

[Rea06] Nathan Reading. Cambrian lattices. Adv. Math., 205(2):313-353, 2006.

[Rea11] Nathan Reading. Noncrossing partitions and the shard intersection order. J. Algebraic Combin., 33(4):483-530, 2011.

[Rea15] Nathan Reading. Noncrossing arc diagrams and canonical join representations. SIAM J. Discrete Math., 29(2):736-750, 2015.

[Rea16a] Nathan Reading. Finite Coxeter groups and the weak order. In Lattice theory: special topics and applications. Vol. 2, pages 489-561. Birkhäuser/Springer, Cham, 2016.

[Rea16b] Nathan Reading. Lattice theory of the poset of regions. In Lattice theory: special topics and applications. Vol. 2, pages 399-487. Birkhäuser/Springer, Cham, 2016.

[RS09] Nathan Reading and David E. Speyer. Cambrian fans. J. Eur. Math. Soc., 11(2):407-447, 2009.

[SS93] Steve Shnider and Shlomo Sternberg. Quantum groups: From coalgebras to Drinfeld algebras. Series in Mathematical Physics. International Press, Cambridge, MA, 1993.

[Tam51] Dov Tamari. Monoides préordonnés et chaînes de Malcev. PhD thesis, Université Paris Sorbonne, 1951.

[Zie98] Günter M. Ziegler. Lectures on Polytopes, volume 152 of Graduate texts in Mathematics. Springer-Verlag, New York, 1998. 